\title{
A Unified Study on L1 over L2 Minimization
}

This paper was downloaded from TechRxiv (https://www.techrxiv.org).

\section{LICENSE}

CC BY 4.0

SUBMISSION DATE / POSTED DATE

31-07-2021 / 05-08-2021

CITATION

Tao, Min; Zhang, Xiao-Ping (2021): A Unified Study on L1 over L2 Minimization. TechRxiv. Preprint. https://doi.org/10.36227/techrxiv.15086277.v1

$\mathrm{DOI}$

10.36227/techrxiv.15086277.v1 


\title{
A Unified Study on $L_{1}$ over $L_{2}$ Minimization
}

\author{
Min Tao and Xiao-Ping Zhang, Fellow, IEEE
}

\begin{abstract}
In this paper, we carry out a unified study for $L_{1}$ over $L_{2}$ sparsity promoting models, which are widely used in the regime of coherent dictionaries for recovering sparse nonnegative/arbitrary signal. First, we provide the exact recovery condition on both the constrained and the unconstrained models for a broad set of signals. Next, we prove the solution existence of these $L_{1} / L_{2}$ models under the assumption that the null space of the measurement matrix satisfies the $s$-spherical section property. Then by deriving an analytical solution for the proximal operator of the $L_{1} / L_{2}$ with nonnegative constraint, we develop a new alternating direction method of multipliers based method $\left(\mathrm{ADMM}_{p}^{+}\right)$to solve the unconstrained model. We establish its global convergence to a d-stationary solution (sharpest stationary) and its local linear convergence under certain conditions. Numerical simulations on two specific applications confirm the superior of $\mathrm{ADMM}_{p}^{+}$over the state-of-the-art methods in sparse recovery. $\mathbf{A D M M}_{p}^{+}$reduces computational time by about $95 \% \sim 99 \%$ while achieving a much higher accuracy compared to commonly used scaled gradient projection method for wavelength misalignment problem.
\end{abstract}

Index Terms-Sparse recovery, the ratio of $L_{1}$ over $L_{2}$, alternating direction method of multipliers, coherent dictionary, d-stationarity

\section{INTRODUCTION}

Compressive sensing (CS) is to seek the sparsest solution from a set of undersampled linear measurements. Mathematically, a fundamental problem in CS can be formulated as a constrained model,

$$
\min _{\mathbf{x} \in \mathcal{X}}\|\mathbf{x}\|_{0}, \text { s.t. } A \mathbf{x}=\mathbf{b}
$$

where $A \in \mathbb{R}^{m \times n}(m \ll n)$ is a sensing matrix, $\|\cdot\|_{0}$ is the $L_{0}$ norm, i.e., the number of nonzero elements. We consider the recovery of nonnegative/arbitrary compressed signal. When the signal is nonnegative, $\mathcal{X}=\mathbb{R}_{+}^{n}$; Otherwise, $\mathcal{X}=\mathbb{R}^{n}$. Unfortunately, the optimization (1) is known to be NP-hard [1]. One common approach is to relax $L_{0}$ norm to $L_{1}$ norm, leading to basis pursuit model [2]. Theoretically, the exact recovery by the $L_{1}$ minimization is guaranteed under the restricted isometry property [3, 4] or null space property [5]. Although the $L_{1}$ minimization technique has been widely used, it is not able to reconstruct the sparsest solutions in

This work of M. Tao is partially supported by National Key Research and Development Program of China (2018AAA0101100), the Natural Science Foundation of China (No. 11971228) and the Jiangsu Provincial National Natural Science Foundation of China (No. BK20181257). The work of X.-P. Zhang is supported by the Natural Sciences and Engineering Research Council of Canada (NSERC), Grant No. RGPIN-2020-04661.

M. Tao is with the Dept. of Mathematics, National Key Laboratory for Novel Software Technology, Nanjing University, Nanjing, 210093, China. Email: taom@nju.edu.cn

X.-P. Zhang is with the Dept. of Electrical, Computer and Biomedical Engineering, Ryerson University, Toronto, ON M5B 2K3, Canada. Email: xzhang@ee.ryerson.ca many applications. As such, various nonconvex minimizations have been developed due to their sharper approximation of $L_{0}$ norm [6-8].

In particular, the ratio of $L_{1} / L_{2}$ norms $\left(L_{1} / L_{2}\right)$, due to its scale-invariant and parameter-free, it has been found a variety applications, such as nonnegative matrix factorization [9], blind deconvolution [10, 11] and limit-angle CT reconstruction [12]. The $L_{1} / L_{2}$ can approximate $L_{0}$ norm very well since it shares the same property of scale-invariant as $L_{0}$ norm. For one-sparse signal case, the $L_{1} / L_{2}$ is the same as the $L_{0}$ norm. Recently, $L_{1} / L_{2}$ minimization model has been empirically verified its efficiency when the sensing matrix is coherent and redundant; see, e.g., [8, 11-17]. More specifically, two types of $L_{1} / L_{2}$ models are commonly used: the constrained and the penalized/unconstrained:

$$
\begin{array}{ll}
\min _{\mathbf{x}} & \frac{\|\mathbf{x}\|_{1}}{\|\mathbf{x}\|_{2}} \\
\text { s.t. } & \mathbf{x} \in \mathcal{H}:=\{\mathbf{x} \in \mathcal{X} \mid A \mathbf{x}=\mathbf{b}\},
\end{array}
$$

and

$$
\min _{\mathbf{x} \in \mathcal{X}} F(\mathbf{x}):=\gamma \frac{\|\mathbf{x}\|_{1}}{\|\mathbf{x}\|_{2}}+\frac{1}{2}\|A \mathbf{x}-\mathbf{b}\|_{2}^{2},
$$

where the compressing matrix $A \in \mathbb{R}^{m \times n}$ and the observation of $\mathbf{b} \in \mathbb{R}^{n}$. The constrained model $(2)$ has been widely used in sparse recovery and MRI reconstruction [12, 14]. However, since the penalized/unconstrained model (3) can tackle both noisy and noiseless observations while (2) can only deal with noiseless data, it turns to be more meaningful to develop efficient and convergent algorithm to solve (3).

First, a few attempts have focused on understanding the equivalence between (1) and (2). A sufficient condition for such an equivalence is called an exact recovery condition. Yin et al. provide an exact recovery condition for (2) when $\mathcal{X}=$ $\mathbb{R}_{+}^{n}$ in [8]. Xu et al. give an exact recovery condition for 2 , when $\mathcal{X}=\mathbb{R}^{n}$ using a geometric characterization of the null space of the matrix $A$. Although its efficiency of successfully recovering the underlying sparse signal via the model (3) has been empirically verified in the existing literature [8, 12, 13, [15], the exact recovery theory is still unknown, Therefore, it motivates us to develop the exact recovery theory on the penalized model (3).

Second, to recover the signal, we need to solve $L_{1}$ over $L_{2}$ minimization models (2) or (3). A first question is whether these models are well-defined. Here, the well-definedness means that the global optimal solution sets are nonempty. Recently, Zeng et al. [17] analyze the existence of global optimal solutions of the constrained model (2) for the case of $\mathcal{X}=\mathbb{R}^{n}$. However, the nonemptyness of the global solution set of the model of $(2)$ with $\mathcal{X}=\mathbb{R}_{+}^{n}$ and the model (3) with $\mathcal{X}=\mathbb{R}_{+}^{n} / \mathbb{R}^{n}$ (i.e., $\mathbb{R}_{+}^{n}$ and $\mathbb{R}^{n}$ ), have not been studied. 
Many early works focus on developing different optimization algorithms for solving $L_{1} / L_{2}$ minimization. To name a few, the scaled gradient projection method [8, 13] for (3) with $\mathcal{X}=\mathbb{R}_{+}^{n}$, as well as the alternating direction method of multiplier [14] for 22) with $\mathcal{X}=\mathbb{R}^{n}$, all lack convergence guarantees. Recently, Zeng et al. [17] apply moving-ballsapproximation algorithms to solve (2) with $\mathcal{X}=\mathbb{R}^{n}$ and prove its global convergence under some conditions. Our previous work [15] proposes an ADMM-based algorthm $\left(\mathrm{ADMM}_{p}\right)$ for solving (3) with $\mathcal{X}=\mathbb{R}^{n}$ with global convergence guarantee. In a short summary, all these algorithms with convergence guarantee are aim to solve the constrained model (2) and the unconstrained model (3) with $\mathcal{X}=\mathbb{R}^{n}$. Although there does exist a few different reformulations for the unconstrained model (3) with $\mathcal{X}=\mathbb{R}_{+}^{n}$, it turns out that most of these for implementing $\mathrm{ADMM}$ result in a violation of convergence guarantee [18-21]. It is still unknown how to solve the model (3) with convergence guarantee where $\mathcal{X}=\mathbb{R}_{+}^{n}$.

In this paper, we aim to carry out a unified theoretical study on (2) and (3). First, we provide a uniformed exact recovery condition for (2) and (3). The exact signal recovery is achieved when the uniformity (i.e., the ratio between the minimum and maximum absolute value of its nonzero entries) of feasible solutions in a neighborhood of the true signal satisfies a certain inequality. As a byproduct, we also show that if any feasible vector cannot be a local minimizer (2) and (3) if the columns of $A$ related the support set of the vector are linearly dependent. Thus, this property serves as a certificate to rule out the stationary solution that is not a local minimizer.

Second, we provide a unified analysis on the existence of global solutions of (2) and (3). We introduce an auxiliary optimization problem and verify that the solution set of (2) is nonempty if the objective function value of (2) is strictly less than that of the auxiliary optimization problem. Furthermore, we prove that the solution set of (3) is nonempty under a similar condition. Then, we illustrate that this sufficient condition can be further guaranteed by the $s$-spherical section property of $\mathcal{N}(A)$ (i.e., the null space of $A$ ).

Third, to design an efficient and convergent algorithm for the penalized model $(3)$ with $\mathcal{X}=\mathbb{R}_{+}^{n}$, we first derive a closedform solution for the proximal operator of $\left(L_{1} / L_{2}\right)^{+}$(i.e., $\left.\left(\frac{\|\mathbf{x}\|_{1}}{\|\mathbf{x}\|_{2}}+\iota_{\mathbb{R}_{+}^{n}}(\mathbf{x})\right)\right)$ and we further develop a practical solver to find one of global solutions. By exploiting the special structure of (3) and using the newly-developed solver of the proximal operator, we propose a specific variable-splitting scheme of ADMM for solving (3) with $\mathcal{X}=\mathbb{R}_{+}^{n}$. We referred it as $\mathrm{ADMM}_{p}^{+}$by incorporating the practical proximal solver. We establish the global convergence of $\mathrm{ADMM}_{p}^{+}$under very mild conditions. Furthermore, we analyze its local linear convergence of $\mathrm{ADMM}_{p}^{+}$under some extra conditions. We test on two specific applications to showcase the superior performance of the proposed approach over the state-of-the-art in sparse nonnegative signal recovery with/without noise. In particular, $\mathrm{ADMM}_{p}^{+}$allows us to significantly reduce the computational time in comparison with SGPM, also leading to a more accurate solution for solving the $\left(L_{1} / L_{2}\right)^{+}$unconstrained model.
The rest of this paper proceeds as follows. We describe the notations and definitions in Section II. In Section III, we provide the exact recovery theory of $L_{1} / L_{2}$ minimization for (2) and (3). We elaborate on the existence of optimal solutions of (2) and (3) in Section IV. In Section V, the proximal operator for $\left(L_{1} / L_{2}\right)^{+}$is derived. Then, we solve the unconstrained $\left(L_{1} / L_{2}\right)^{+}$model $(3)$ via ADMM, where its global convergence and local linear convergence rate are established. Section VI devotes extensive experiments to showcase the superior performance of the proposed approach in sparse recovery. Conclusions are given in Section VII.

\section{PRELIMINARY}

We use a bold letter to denote a vector, e.g., $\mathbf{x} \in \mathbb{R}^{n}$, and $x_{i}$ denotes the $i$-th entry of $\mathbf{x}$ and $\|\mathbf{x}\|_{0}$ represents its zero norm and $|\mathbf{x}|$ represents the vector with the absolute value for each entry of $\mathbf{x}$, and $\|\mathbf{x}\|_{2}$ denotes its 2-norm, i.e., $\|\mathbf{x}\|_{2}=$ $\left(\sum_{i=1}^{n} x_{i}^{2}\right)^{1 / 2} ;\|\mathbf{x}\|_{p}(0<p<1)$ denotes its $p$-norm, i.e., $\|\mathbf{x}\|_{p}=\left(\sum_{i=1}^{n} x_{i}^{p}\right)^{1 / p}$. We denote $\|\cdot\|_{2}$ as $\|\cdot\|$ when there is no ambiguity. $I_{n}$ is $n \times n$ identity matrix, and $\odot$ to present the componentwise product. We define $[n]:=\{1,2, \ldots, n\}$ as an index set up to $n$ and given an index set $\mathcal{D} \subseteq[n]$, we use $\sharp(\mathcal{D})$ and $\mathcal{D}^{c}$ to present the cardinality of $\mathcal{D}$ and its complementary set. Give a matrix $A \in \mathbb{R}^{m \times n}$, we denote $A \mathcal{X}=\{\mathbf{y} \mid \mathbf{y}=A \mathbf{x}, \mathbf{x} \in \mathcal{X}\}$ and $\operatorname{refer} \operatorname{Proj}_{(A \mathcal{X})}(\cdot)$ to the projection onto the closed set of $A \mathcal{X}$. For a closed set $\mathcal{S} \in \mathbb{R}^{n}$, we use the notation $\iota_{\mathcal{S}}(\mathrm{x})$ to represent the indicator function of the set $\mathcal{S}$. Given a matrix $A \in \mathbb{R}^{m \times n}$ or a vector $\mathbf{x} \in \mathbb{R}^{n}$ and an index set $\Lambda \subseteq[n]$, we use $A_{\Lambda},\left.\mathbf{x}\right|_{\Lambda}$ to denote $A[:, i]_{i \in \Lambda}$ and a subvector of $\mathrm{x}$ with entries in $\Lambda$, respectively. $\mathcal{N}(A)$ denotes the null space of $A$ and $r(A)$ denotes the rank of $A$. Given $\epsilon>0$, we use $\mathcal{B}_{\epsilon}(\hat{\mathbf{x}})$ and $\breve{\mathcal{B}}_{\epsilon}(\hat{\mathbf{x}})$ to denote the open ball of $\{\mathbf{x} \mid\|\mathbf{x}-\hat{\mathbf{x}}\|<\epsilon\}$ and the open ball without the center, respectively. If $\hat{\mathbf{x}}=0$. we denote $\mathcal{B}_{\epsilon}(\hat{\mathbf{x}})$ as $\mathcal{B}_{\epsilon}$ for conciseness. Given two sets of $\mathcal{A}$ and $\mathcal{B}$, we use the notation $\mathcal{A} \backslash \mathcal{B}$ to present product of $\mathcal{A}$ and the complement of $\mathcal{B}$. The notation of $\mathbb{R}_{+}^{n} / \mathbb{R}^{n}$ means $\mathbb{R}_{+}^{n}$ and $\mathbb{R}^{n}$. We define the subgradient of the $\|\cdot\|_{1}$ norm as

$$
(\operatorname{sign}(\mathbf{x}))_{i}:= \begin{cases}1 & x_{i}>0 \\ {[-1,1]} & x_{i}=0 \\ -1 & x_{i}<0\end{cases}
$$

In view of [22, Proposition 2.3.14], it holds that for $\mathbf{x} \neq \mathbf{0}$, $\partial\left(\frac{\|\mathbf{x}\|_{1}}{\|\mathbf{x}\|_{2}}\right)=\frac{\partial\|\mathbf{x}\|_{1}}{\|\mathbf{x}\|_{2}}-\frac{\|\mathbf{x}\|_{1}}{\|\mathbf{x}\|_{2}^{3}} \mathbf{x}$. Note $\partial \iota \mathcal{X}(\mathbf{x})=\{\mathbf{0}\}$ if $\mathcal{X}=$ $\mathbb{R}^{n}$, and $\partial_{\iota \mathcal{X}}(\mathbf{x})=\left\{\mathbf{d} \mid \mathbf{d}_{\Lambda}=\mathbf{0}, \mathbf{d}_{\Lambda^{c}} \leq \mathbf{0}, \Lambda=\operatorname{supp}(\mathbf{x})\right\}$ if $\mathcal{X}=\mathbb{R}_{+}^{n}$. Throughout the paper, we assume that $\mathbf{b} \neq 0$ and $\mathcal{H} \neq \phi$. By defining $\frac{\|\mathbf{0}\|_{1}}{\|\mathbf{0}\|_{2}}=1$, the objective function of $\sqrt{2}$ is lower semi-continuous over $\mathcal{X}$. Given two integers of $m$ and $n(m<n)$, we use the notation $C_{n}^{m}$ to denote the number of the ways of taking $m$ from $n$.

For nonconvex and nonsmooth program, there are various stationary concepts [23] and d-stationary is arguably the sharpest kind among them [24, 25]. A point $\overline{\mathbf{x}}(\neq \mathbf{0}) \in \mathcal{X}$ is called a d-stationary point to $(3)$, if it satisfies

$$
F^{\prime}\left(\overline{\mathbf{x}} ; \mathbf{x}^{\prime}-\overline{\mathbf{x}}\right) \geq 0 . \quad \forall \mathbf{x}^{\prime} \in \mathcal{X}
$$


where $F^{\prime}\left(\overline{\mathbf{x}} ; \mathbf{x}^{\prime}-\overline{\mathbf{x}}\right)$ is the directional derivative of $F(\cdot)$. According to [22, Definition 2.3.4] and [23, Fact 5], $\overline{\mathbf{x}}(\neq \mathbf{0}) \in \mathcal{X}$ is a d-stationary point of (3) if and only if

$$
\begin{array}{r}
\left\langle\mathbf{x}^{\prime}-\overline{\mathbf{x}}, \gamma\left(\frac{\overline{\mathbf{p}}}{\|\overline{\mathbf{x}}\|_{2}}-\frac{\|\overline{\mathbf{x}}\|_{1}}{\|\overline{\mathbf{x}}\|_{2}^{3}} \overline{\mathbf{x}}\right)+A^{\top}(A \overline{\mathbf{x}}-\mathbf{b})\right\rangle \geq 0, \\
\forall \mathbf{x}^{\prime} \in \mathcal{X},
\end{array}
$$

where $\overline{\mathbf{p}} \in \operatorname{sign}(\overline{\mathbf{x}})$.

Next, we review on KL property and KL exponent [26] which are widely used in convergence and convergence rate analysis.

Definition 1. We say a proper closed function $h: \mathbb{R}^{n} \rightarrow$ $(-\infty,+\infty]$ satisfies $K L$ property at a point $\hat{\mathbf{x}} \in$ domoh if there exist a constant $\alpha \in(0, \infty]$, a neighborhood $U$ of $\hat{\mathbf{x}}$, and a continuous concave function $\phi:[0, \nu) \rightarrow[0, \infty)$ with $\phi(0)=0$ such that

(i) $\phi$ is continuously differentiable on $(0, \nu)$ with $\phi^{\prime}>0$ on $(0, \nu)$

(ii) for every $\mathrm{x} \in U$ with $h(\hat{\mathbf{x}})<h(\mathbf{x})<h(\hat{\mathbf{x}})+\nu$, it holds that $\phi^{\prime}(h(\mathbf{x})-h(\hat{\mathbf{x}})) \operatorname{dist}(\mathbf{0}, \partial h(\mathbf{x})) \geq 1$.

Definition 2. For a proper closed function $h$ satisfying the $K L$ property at $\hat{\mathbf{x}} \in$ dom $\partial h$, if the corresponding function $\phi$ can be chosen as $\phi(s)=a_{0} s^{1-\theta}$ for some $a_{0}>0$ and $\theta \in[0,1)$, i.e., there exist $c, \varepsilon>0$ and $\nu \in(0,+\infty]$ so that

$$
\operatorname{dist}(\mathbf{0}, \partial h(\mathbf{x})) \geq c(h(\mathbf{x})-h(\hat{\mathbf{x}}))^{\theta}
$$

whenever $\|\mathbf{x}-\hat{\mathbf{x}}\| \leq \varepsilon$ and $h(\hat{\mathbf{x}})<h(\mathbf{x})<h(\hat{\mathbf{x}})+\nu$, then we say $h$ has the KL property at $\hat{\mathbf{x}}$ with an exponent of $\theta$. If $h$ is a $K L$ function and has the same exponent $\theta$ at any $\hat{\mathbf{x}} \in d o m \partial h$, then we say that $h$ is a KL function with exponent of $\theta$.

We can define the KL exponent of $h$ restricted on a closed set of $\Xi$ when Definition 2 holds on the set $\Xi$.

Next, we review the concept of locally sparse set [8] and introduce the uniformity of a vector $\mathbf{x}$.

Definition 3. $\mathrm{x} \in \mathcal{H}$ is called locally sparse if $\nexists \mathbf{y} \in \mathcal{H} \backslash\{\mathbf{x}\}$ $(\mathcal{H}$ defined in (2)) such that

$$
\operatorname{supp}(\mathbf{y}) \subseteq \operatorname{supp}(\mathbf{x})
$$

Denote by $\mathcal{H}_{L}=\{\mathbf{x} \in \mathcal{H} \mid \mathbf{x}$ is locally sparse $\}$.

Definition 4. The uniformity of $\mathbf{x}, \kappa(\mathbf{x})$ is the ratio between the smallest nonzero absolute entry and the largest one in the sense of absolute value, i.e.

$$
0<\kappa(\mathbf{x}):=\frac{\min _{i \in \operatorname{supp}(\mathbf{x})}\left|x_{i}\right|}{\max _{i \in \operatorname{supp}(\mathbf{x})}\left|x_{i}\right|} \leq 1 .
$$

Our analysis on the existence of globally optimal solutions is based on the spherical section property (SSP) [17, 27, 28].

Definition 5. Let $m, n$ be two positive integers such that $m<n$. Let $V$ be an $(n-m)$-dimensional subspace of $\mathbb{R}^{n}$ and $s$ be a positive integer. We say that $V$ has the $s$-spherical section property if

$$
\inf _{\mathbf{v} \in V \backslash\{0\}} \frac{\|\mathbf{v}\|_{1}}{\|\mathbf{v}\|_{2}} \geq \sqrt{\frac{m}{s}}
$$

As illustrated in [17, 28], if $A \in \mathbb{R}^{m \times n}(m<n)$ is a random matrix with i.i.d. standard Gaussian entries, then its nullspace has the $s$-spherical section property with high probability.

\section{EXACT RECOVERY OF $L_{1} / L_{2}$ MINIMIZATION}

\section{A. Sparse property of local minimizers}

In this subsection, we demonstrate that the sparsity of the local minimizers of (2) and (3) in the sense that minimizing $L_{1} / L_{2}$ or $\left(L_{1} / L_{2}\right)^{+}$only extract linearly independent columns from the sensing matrix $A$. Second, we further explore the property of any local minimizer of (2) and (3) in Corollary 1

Theorem 1. Let $\mathrm{x}^{*}\left(\mathrm{x}^{*} \neq \mathbf{0}\right)$ be a local minimizer of the constrained problem (2) and $\Lambda^{*}=\operatorname{supp}\left(\mathrm{x}^{*}\right)$. Then, $\left(A_{\Lambda^{*}}\right)^{\top}\left(A_{\Lambda^{*}}\right) \succ \mathbf{0}$.

Proof: We divide into two cases to verify.

Case 1: $\mathcal{X}=\mathbb{R}^{n}$. Let $\mathrm{x}^{*}$ be a local minimizer of the constrained model (2). We use contradiction to show the assertion. Suppose the columns of $A_{\Lambda^{*}}$ are linearly dependent; then there exists $\mathbf{v} \neq \mathbf{0}$ and $\mathbf{v} \in \mathcal{N}(A)$ such that $\operatorname{supp}(\mathbf{v}) \subseteq \Lambda^{*}$. For any fixed neighborhood $\mathcal{B}_{r}\left(\mathbf{x}^{*}\right)$ of $\mathbf{x}^{*}$, we scale $\mathbf{v}$ so that $\|\mathbf{v}\|_{2}<\min \left\{\min _{i \in \Lambda^{*}}\left|x_{i}^{*}\right|, r\right\}$. Consider two feasible vectors in $\mathcal{B}_{r}\left(\mathbf{x}^{*}\right), \hat{\mathbf{x}}=\mathbf{x}^{*}+\mathbf{v}$ and $\check{\mathbf{x}}=\mathbf{x}^{*}-\mathbf{v}$. Since $\operatorname{supp}(\mathbf{v}) \subseteq \Lambda^{*}$, we have $\operatorname{supp}(\hat{\mathbf{x}}) \subseteq \Lambda^{*}$ and $\operatorname{supp}(\check{\mathbf{x}}) \subseteq \Lambda^{*}$. Since for any $i \in \Lambda^{*}$, $\left|x_{i}^{*}\right| \pm \operatorname{sign}\left(x_{i}^{*}\right) v_{i} \geq \min _{i \in \Lambda^{*}}\left|x_{i}^{*}\right|-\|\mathbf{v}\|_{2}>0, \forall i \in \Lambda^{*}$. Thus, $\left(\mathbf{x}^{*} \pm \mathbf{v}\right)_{i}=\operatorname{sign}\left(x_{i}^{*}\right)\left(\left|x_{i}^{*}\right| \pm \operatorname{sign}\left(x_{i}^{*}\right) v_{i}\right)$ for any $i \in \Lambda^{*}$. It implies that $\mathbf{x}^{*}, \hat{\mathbf{x}}$ and $\check{\mathbf{x}}$ are located in the same octant. Consequently,

$$
\left\|\mathbf{x}^{*}\right\|_{1}=\frac{1}{2}\left(\|\hat{\mathbf{x}}\|_{1}+\|\check{\mathbf{x}}\|_{1}\right) \text { and }\left\|\mathbf{x}^{*}\right\|_{2}<\frac{1}{2}\left(\|\hat{\mathbf{x}}\|_{2}+\|\check{\mathbf{x}}\|_{2}\right),
$$

where the inequality is due to the facts of $A \hat{\mathbf{x}}=\mathbf{b}$ and $A \check{\mathbf{x}}=$ b. Finally, it yields that

$$
\frac{\left\|\mathbf{x}^{*}\right\|_{1}}{\left\|\mathbf{x}^{*}\right\|_{2}}>\min \left\{\frac{\|\hat{\mathbf{x}}\|_{1}}{\|\hat{\mathbf{x}}\|_{2}}, \frac{\|\check{\mathbf{x}}\|_{1}}{\|\check{\mathbf{x}}\|_{2}}\right\},
$$

which contradicts to the fact that $\mathrm{x}^{*}$ is a local minimizer. Case 2: $\mathcal{X}=\mathbb{R}_{+}^{n}$. The proof is similar to Case 1 , thus omitted here.

Next, we show that the conclusion of Theorem 1 also holds for the unconstrained model.

Theorem 2. Let $\mathrm{x}^{*}$ be a local minimizer of the unconstrained problem (3) and $\Lambda^{*}=\operatorname{supp}\left(\mathrm{x}^{*}\right)$. Then,

$$
\left(A_{\Lambda^{*}}\right)^{\top}\left(A_{\Lambda^{*}}\right) \succ \mathbf{0} \text {. }
$$

Proof: First, we show that $\mathrm{x}^{*}$ is also a local minimizer of the constrained problem (2) where $\mathbf{b}:=A \mathbf{x}^{*}$. Suppose not. Then, for any $r>0$, there exists $\mathbf{x}_{r} \in \mathcal{B}_{r}\left(\mathbf{x}^{*}\right) \cap \mathcal{X}$ such that $A \mathbf{x}_{r}=A \mathbf{x}^{*}$ and $\frac{\left\|\mathbf{x}_{r}\right\|_{1}}{\left\|\mathbf{x}_{r}\right\|_{2}}<\frac{\left\|\mathbf{x}^{*}\right\|_{1}}{\left\|\mathbf{x}^{*}\right\|_{2}}$. It further implies that $F\left(\mathbf{x}_{r}\right)<F\left(\mathbf{x}^{*}\right)$ where $F$ is defined in 3 , which contradicts to that $\mathrm{x}^{*}$ is a local minimizer of (3). Therefore, $\mathrm{x}^{*}$ is a local minimizer of (2) where $\mathbf{b}:=A \mathbf{x}^{*}$. By invoking Theorem 1 . the conclusion follows directly.

Remark 1. From Theorems 1 and 2 , we see that if a computed solution $\mathbf{x}$ from the model (2) or (3) fails to extract linearly 
independent columns from the sensing matrix $A$. Then, $\mathbf{x}$ cannot be a local minimizer.

The next lemma present a sufficient and necessary condition for characterizing $\mathbf{x} \in \mathcal{H}_{L}$ which turns to be checkable.

Lemma 1. $\mathrm{x} \in \mathcal{H}_{L}$ if and only if $A_{\Lambda}^{\top} A_{\Lambda} \succ \mathbf{0}$ where $\Lambda=$ $\operatorname{supp}(\mathbf{x})$.

Proof: First, we verify the direction of "only if". Denote $\alpha=\sharp(\Lambda)$. Suppose that $\mathbf{x} \in H_{L}$, we use contradiction to show that $A_{\Lambda}^{\top} A_{\Lambda} \succ \mathbf{0}$. Suppose not. It implies that there exists a vector $\mathbf{v} \in \mathbb{R}^{\alpha}(\mathbf{v} \neq \mathbf{0})$ such that $\mathbf{v} \in \mathcal{N}\left(A_{\Lambda}\right)$ and define another vector

$$
\tilde{\mathbf{v}}= \begin{cases}v_{i} & i \in \Lambda \\ 0 & i \notin \Lambda,\end{cases}
$$

where $\tilde{\mathbf{v}} \in \mathbb{R}^{n}$. We define an index set: $\Lambda_{\tilde{\mathbf{v}}}:=\operatorname{supp}(\tilde{\mathbf{v}})$. Note that $\operatorname{supp}(\tilde{\mathbf{v}}) \subseteq \Lambda$. Let $\zeta:=\min _{i \in \Lambda_{\tilde{\mathbf{v}}}}\left|\frac{x_{i}}{\tilde{v}_{i}}\right|>0$ and $\tilde{i} \in \arg \min _{i \in \Lambda_{\tilde{\mathbf{v}}}}\left|\frac{x_{i}}{\tilde{v}_{i}}\right|$. Next, we define the vector: $\tilde{\mathbf{x}}=$ $\mathbf{x}-\operatorname{sign}\left(x_{i} \tilde{v}_{i}\right) \zeta \tilde{\mathbf{v}}$. Then, $\tilde{\mathbf{x}} \in \mathcal{H}$, and it is much sparser than $\mathbf{x}$ and $\operatorname{supp}(\tilde{\mathbf{x}}) \subseteq \operatorname{supp}(\mathbf{x})$ which contradicts to $\mathbf{x} \in$ $\mathcal{H}_{L}$. Second, for the direction of "if", we use contradiction. Suppose not. There exists another feasible solution $\mathbf{y}$ such that $\operatorname{supp}(\mathbf{y}) \subseteq \operatorname{supp}(\mathbf{x})$, and $A \mathbf{y}=\mathbf{b}, \mathbf{y} \in \mathcal{X}$. Then, we define $\mathbf{v}:=\mathbf{y}-\mathbf{x}(\neq \mathbf{0}) \in \mathcal{N}\left(A_{\Lambda}\right)$ which contradicts to $\left(A_{\Lambda}\right)^{\top}\left(A_{\Lambda}\right) \succ \mathbf{0}$ which contradicts to $A_{\Lambda}^{\top} A_{\Lambda} \succ \mathbf{0}$. Thus, the conclusion follows directly.

Combining Theorems 1 and 2 and Lemma 1, we conclude the following corollary.

Corollary 1. We have these facts hold:

(i) Suppose that $\operatorname{rank}(A)=m$ and $\mathrm{x}^{*}$ is a local minimizer of (2) and (3), the sparsity of $\mathrm{x}^{*}$ is at most $m$.

(ii) Both of the models (2) and (3) have finite local minimizers.

(iii) If $\mathrm{x}^{*}$ is a local minimizer of (2) or (3), then $\mathrm{x}^{*} \in \mathcal{H}_{L}$ where $\mathcal{H}_{L}$ is defined in Definition 3

Proof: Obviously, the assertions (i) and (ii) are true. (iii) Suppose that $\mathrm{x}^{*}$ is a local minimizer of (2) or (3), it leads to $\left(A_{\Lambda^{*}}\right)^{\top}\left(A_{\Lambda^{*}}\right) \succ \mathbf{0}$ due to Theorem 1 or 2 . Invoking Lemma 1. the conclusion follows directly.

\section{B. Exact recovery theory}

In this subsection, we establish the exact recovery condition on (2) with $\mathcal{X}=\mathbb{R}^{n} / \mathbb{R}_{+}^{n}$ in Theorems 3 and 4 under two different while equivalent assumptions. Moreover, the latter turns to be more checkable. Next, we provide the exact recovery condition of 3 for $\mathcal{X}=\mathbb{R}^{n} / \mathbb{R}_{+}^{n}$ in Theorem 5 .

Lemma 2. $\forall \mathbf{x} \in \mathcal{H}_{L}$ where $\mathcal{H}_{L}$ is defined in Definition 3 . $\exists \delta>0$ such that $\forall \mathbf{y} \in \mathcal{H} \cap \check{\mathcal{B}}_{\delta}(\mathbf{x})$ ( $\mathcal{H}$ defined in (2)), we have $\operatorname{supp}(\mathbf{x}) \subseteq \operatorname{supp}(\mathbf{y})$.

Proof: Choosing $\delta:=\min _{i \in \operatorname{supp}(\mathbf{x})}\left|x_{i}\right|$. For any $0<\| \mathbf{y}-$ $\mathbf{x} \|<\delta$, we have that

$$
\left|x_{i}\right| \neq 0 \Rightarrow\left|y_{i}\right| \neq 0 \text {. }
$$

Consequently, $\operatorname{supp}(\mathbf{x}) \subseteq \operatorname{supp}(\mathbf{y})$.
From Item (iii) of Corollary 1, we see that any local/global minimizer must be locally sparsest feasible solution which also plays a key role in the coming analysis. The following lemma presents a lower bound for the ratio of $L_{1}$ and $L_{2}$ norms.

Lemma 3. Let $\mathbf{x}=\left[x_{1}, \cdots, x_{j-1}, x_{j}, x_{j+1}, \cdots, x_{n}\right]^{\top}$. Define two vectors

$$
\begin{aligned}
& \mathbf{x}_{L}=\left[\left|x_{1}\right|, \cdots,\left|x_{j-1}\right|, \kappa(\mathbf{x}),\left|x_{j+1}\right|, \cdots,\left|x_{n}\right|\right]^{\top}, \\
& \mathbf{x}_{U}=\left[\left|x_{1}\right|, \cdots,\left|x_{j-1}\right|, 1,\left|x_{j+1}\right|, \cdots,\left|x_{n}\right|\right]^{\top},
\end{aligned}
$$

where $\kappa(\mathbf{x})$ is defined in Definition 4 . Then, we have

$$
\frac{\|\mathbf{x}\|_{1}}{\|\mathbf{x}\|_{2}} \geq \min \left\{\frac{\left\|\mathbf{x}_{L}\right\|_{1}}{\left\|\mathbf{x}_{L}\right\|_{2}}, \frac{\left\|\mathbf{x}_{U}\right\|_{1}}{\left\|\mathbf{x}_{U}\right\|_{2}}\right\}
$$

Proof: By defining $\tilde{\mathbf{x}}:=|\mathbf{x}|$, we note that $\frac{\|\mathbf{x}\|_{1}}{\|\mathbf{x}\|_{2}}=\frac{\|\tilde{\mathbf{x}}\|_{1}}{\|\tilde{\mathbf{x}}\|_{2}}$. There exists a constant $\alpha \in[0,1]$ such that $\tilde{x}_{j}=\alpha \kappa(\mathbf{x})+(1-$ $\alpha$ ), which implies that $\tilde{\mathbf{x}}=\alpha \mathbf{x}_{L}+(1-\alpha) \mathbf{x}_{U}$. Therefore, the assertion follows directly.

We are now in the stage to provide our results.

Theorem 3. If $\mathbf{x}_{0}$ uniquely solves (1) and $\left\|\mathbf{x}_{0}\right\|_{0}=s$ and if

$$
\kappa(\mathbf{x})>\frac{\left(\sqrt{\|\mathbf{x}\|_{0}}-\sqrt{\|\mathbf{x}\|_{0}-s}\right)^{2}}{s}, \forall \mathbf{x} \in \mathcal{H}_{L} \backslash\left\{\mathbf{x}_{0}\right\},
$$

then $\mathrm{x}_{0}$ also uniquely solve (2).

Proof: For the case of $\mathcal{X}=\mathbb{R}_{+}^{n}$, it has been proved in [8. Theorem III.2] by noting that $\frac{\sqrt{\|\mathbf{x}\|_{0}}-\sqrt{\|\mathbf{x}\|_{0}-s}}{\sqrt{\|\mathbf{x}\|_{0}}+\sqrt{\|\mathbf{x}\|_{0}-s}}=$ $\frac{\left(\sqrt{\|\mathbf{x}\|_{0}}-\sqrt{\|\mathbf{x}\|_{0}-s}\right)^{2}}{s}$. For the case of $\mathcal{X}=\mathbb{R}^{n}$, the proof is similar to the case of $\mathcal{X}=\mathbb{R}_{+}^{n}$, thus omitted here.

Based on the above theorem, we present another exact recovery condition. It turns to be much more easily checkable for both $\mathcal{X}=\mathbb{R}^{n} / \mathbb{R}_{+}^{n}$. Next, we define the following set:

$$
\tilde{\mathcal{F}}:=\left\{\mathbf{x} \in \mathcal{H} \mid \Lambda=\operatorname{supp}(\mathbf{x}), A_{\Lambda}^{\top} A_{\Lambda} \succ \mathbf{0}\right\},
$$

where $\mathcal{H}$ is defined in 2 .

Theorem 4. If $\mathbf{x}_{0}$ uniquely solves (1) and $\left\|\mathbf{x}_{0}\right\|_{0}=s$ and if

$$
\kappa(\mathbf{x})>\frac{\left(\sqrt{\|\mathbf{x}\|_{0}}-\sqrt{\|\mathbf{x}\|_{0}-s}\right)^{2}}{s}, \forall \mathbf{x} \in \tilde{\mathcal{F}} \backslash\left\{\mathbf{x}_{0}\right\},
$$

then $\mathrm{x}_{0}$ also uniquely solve (2).

Proof: It follows directly from Lemma 1 and Theorem 3 directly.

Similarly, we can obtain the following exact recovery theory on the unconstrained model (3).

Theorem 5. If $\mathbf{x}_{0}$ uniquely solves (1) and $\left\|\mathbf{x}_{0}\right\|_{0}=s$ and if

$$
\kappa(\mathbf{x})>\frac{\left(\sqrt{\|\mathbf{x}\|_{0}}-\sqrt{\|\mathbf{x}\|_{0}-s}\right)^{2}}{s}, \forall \mathbf{x} \in \tilde{\mathcal{F}} \backslash\left\{\mathbf{x}_{0}\right\},
$$

then $\mathbf{x}_{0}$ also uniquely solve (3).

Proof: Since $\frac{\left\|\mathbf{x}_{0}\right\|_{1}}{\left\|\mathbf{x}_{0}\right\|_{2}}<\frac{\|\mathbf{x}\|_{1}}{\|\mathbf{x}\|_{2}}, \forall \mathbf{x} \in \tilde{\mathcal{F}} \backslash\left\{\mathbf{x}_{0}\right\}$, and $A \mathbf{x}_{0}=$ $\mathbf{b}$, it leads to $F\left(\mathbf{x}_{0}\right)<F(\mathbf{x}), \forall \mathbf{x} \in \tilde{\mathcal{F}} \backslash\left\{\mathbf{x}_{0}\right\}$. Thus, the conclusion follows immediately. 
Now, we have provided a uniform exact recovery condition of (8) for both (2) and (3). This sufficient condition says that the uniformity of any feasible vector in $\tilde{\mathcal{F}} \backslash\left\{\mathbf{x}_{0}\right\}$ is required to large than a threshold which is related to its zero norm of the feasible vector and $\left\|\mathbf{x}_{0}\right\|_{0}$.

Remark 2. The exact recovery condition (8) holds for a broad set of cases. For example, it is easy to show that the inequality of (8) is satisfied in the following two cases: (1) the rank $A$ is one; Or (2) the feasible signals are with all entries $0, \pm 1$.

In general, the set $\tilde{\mathcal{F}}$ contains at most a finite number of vectors. Suppose that the rank of $A$ is $r(A)$, then the number of the vectors in the set of $\tilde{\mathcal{F}}$ is at most $C_{n}^{1}+C_{n}^{2}+\cdots+C_{n}^{r(A)}$. When the matrix $A$ is highly coherent, i.e.,

$$
\mu(A):=\max _{1 \leq i<j \leq n} \frac{\mathbf{a}_{i}^{\top} \mathbf{a}_{j}}{\left\|\mathbf{a}_{i}\right\|\left\|\mathbf{a}_{j}\right\|} \sim 1,
$$

it means $r(A)$ might be small. Therefore, to ensure the exact recovery condition, we only need to check a finite number of feasible vectors of satisfying (8) and the number of the testing vectors is insignificant especially when the rank of $A$ is very small.

\section{EXISTENCE OF OPTIMAL SOLUTIONS}

In this section, we explore the conditions to guarantee the existence of global solutions of (2) and (3). First, we verify that the solution set of (2) is nonempty if the optimal value of (2) is less than that of the auxiliary problem $(9)$. Second, we prove that the solution set of (3) is nonempty when the objective function value of newly-introduced constrained model which closely relate to (3) is less than (9). Finally, we further show that these sufficient conditions to guarantee the solution set nonempty can be ensured by the null space of the measurement matrix satisfies the $s$-spherical section property.

Our analysis is inspired by [17] and introduce the following auxiliary problem:

$$
\begin{array}{ll}
f_{d c}^{*}:= & \inf _{\mathbf{d}} \frac{\|\mathbf{d}\|_{1}}{\|\mathbf{d}\|_{2}} \\
\text { s.t. } & \mathbf{d} \in \mathcal{F}_{0}:=\{\mathbf{d} \mid A \mathbf{d}=\mathbf{0}, \mathbf{d} \in \mathcal{X}, \mathbf{d} \neq \mathbf{0}\} .
\end{array}
$$

For analysis convenience, we denote the optimal value of (2) as $f_{p c}^{*}$ and $f_{p c}^{*}<+\infty$. We recall $\left\{\mathbf{x}^{k}\right\}$ is a minimizing sequence of 22 if $\mathbf{x}^{k} \in \mathcal{H}$ for each $k$ and $\lim _{k \rightarrow \infty} \frac{\left\|\mathbf{x}^{k}\right\|_{1}}{\left\|\mathbf{x}^{k}\right\|_{2}}=f_{p c}^{*}$. Therefore we only need to characterize the existence of unbounded minimizing sequence. Before that, we provide a sufficient condition to guarantee the solution set of 9 nonempty.

Lemma 4. Let $f_{d c}^{*}$ be defined in (9). Assume that $\mathcal{N}(A) \cap \mathcal{X} \neq$ $\{0\}$. Then, $f_{d c}^{*}<+\infty$ and the solution set of 9 is nonempty.

Proof: First, the feasible set of 9 is nonempty due to $\mathcal{N}(A) \cap \mathcal{X} \neq\{\mathbf{0}\}$. The objective function value is lower bounded, i.e., $\frac{\|\mathbf{d}\|_{1}}{\|\mathbf{d}\|_{2}} \geq 1$. Suppose that there exists a minimizing sequence $\left\{\tilde{\mathbf{d}}^{k}\right\}$ of 90 that is unbounded with $\frac{\left\|\tilde{\mathbf{d}}^{k}\right\|_{1}}{\left\|\tilde{\mathbf{d}}^{k}\right\|_{2}} \rightarrow f_{d c}^{*}$ as $k \rightarrow+\infty$. Thus, $f_{d c}^{*}<+\infty$. Consequently, by defining $\mathbf{d}^{k}=\frac{\tilde{\mathbf{d}}^{k}}{\left\|\tilde{\mathbf{d}}^{k}\right\|_{2}}$, the sequence $\left\{\mathbf{d}^{k}\right\}$ is satisfying $\mathbf{d}^{k} \in \mathcal{F}_{0}$ and $\lim _{k \rightarrow+\infty} \frac{\left\|\mathbf{d}^{k}\right\|_{1}}{\left\|\mathbf{d}^{k}\right\|_{2}}=f_{d c}^{*}$. Since the sequence of $\left\{\mathbf{d}^{k}\right\}$ is bounded, it has one accumulation point $\mathbf{d}^{*} \in \mathcal{F}_{0}$. Therefore, the solution value of (9) is attainable by $\mathbf{d}^{*}$.

The following proposition shows that the optimal value of (2) is upper bounded by that of (9) when the feasible sets of both (2) and (9) are nonempty.

Proposition 1. Suppose that $\mathcal{N}(A) \cap \mathcal{X} \neq\{\mathbf{0}\}$. Then, $f_{p c}^{*} \leq$ $f_{d c}^{*}$

Proof: Since $\mathcal{N}(A) \cap \mathcal{X} \neq\{\mathbf{0}\}$, it leads to $\mathcal{F}_{0} \neq \phi$. For any $\hat{\mathbf{x}} \in \mathcal{H}$ and any $\hat{\mathbf{d}} \in \mathcal{F}_{0}$, we have $\hat{\mathbf{x}}+\tau \hat{\mathbf{d}} \in \mathcal{H}$ where $\tau>0$. Thus, it leads to $f_{p c}^{*}=\inf _{\mathbf{x} \in \mathcal{H}} \frac{\|\mathbf{x}\|_{1}}{\|\mathbf{x}\|_{2}} \leq \frac{\|\hat{\mathbf{x}}+\tau \hat{\mathbf{d}}\|_{1}}{\|\hat{\mathbf{x}}+\tau \hat{\mathbf{d}}\|_{2}}$. On the other hand, we have that $\lim _{\tau \rightarrow+\infty} \frac{\|\hat{\mathbf{x}}+\tau \hat{\mathbf{d}}\|_{1}}{\|\hat{\mathbf{x}}+\tau \hat{\mathbf{d}}\|_{2}}=$ $\lim _{\tau \rightarrow+\infty} \frac{\|\hat{\mathbf{x}} / \tau+\hat{\mathbf{d}}\|_{1}}{\|\hat{\mathbf{x}} / \tau+\hat{\mathbf{d}}\|_{2}}=\frac{\|\hat{\mathbf{d}}\|_{1}}{\|\hat{\mathbf{d}}\|_{2}}$. Consequently, for any $\hat{\mathbf{d}} \in \mathcal{F}_{0}$, it yields that $f_{p c}^{*} \leq \frac{\|\hat{\mathbf{d}}\|_{1}}{\|\hat{\mathbf{d}}\|_{2}}$. By taking infimum on both sides of the above inequality with respect to $\hat{\mathbf{d}}$, we have the desired inequality.

Theorem 6. Assume that $\mathcal{N}(A) \cap \mathcal{X} \neq\{\mathbf{0}\}$. Consider (2) and (9). Then, $f_{p c}^{*}=f_{d c}^{*}$ if and only if there exists a minimizing sequence of (2) that is unbounded.

Proof: For the case of $\mathcal{X}=\mathbb{R}^{n}$, it has been proved in [17, Lemma 3.3] that $f_{p c}^{*}=f_{d c}^{*}$ if and only if there exists a minimizing sequence of (2) that is unbounded. The proof for the case of $\mathcal{X}=\mathbb{R}_{+}^{n}$ is similar to the case of $\mathcal{X}=\mathbb{R}^{n}$, and thus omitted here.

Corollary 2. Suppose that $\mathcal{N}(A) \cap \mathcal{X} \neq\{\mathbf{0}\}$. Consider and (9). If $f_{p c}^{*}<f_{d c}^{*}$, the solution set of (2) is nonempty.

Proof: It follows directly by combining Theorem 6 and Proposition 1 .

Next, we analyze the existence of the global solution of the penalized model (3). In doing so, we introduce a constrained problem parameterized by the vector $\mathbf{c}$ :

$$
\begin{array}{ll}
\min _{\mathbf{x}} & \frac{\|\mathbf{x}\|_{1}}{\|\mathbf{x}\|_{2}} \\
\text { s.t. } & A \mathbf{x}=\mathbf{c}, \\
& \mathbf{x} \in \mathcal{X},
\end{array}
$$

and denote the optimal value of 10 as $f_{p c}^{*}(\mathbf{c})$ and $f_{p c}^{*}(\mathbf{c})<$ $+\infty$ for any $\mathbf{c} \in A \mathcal{X}$.

Theorem 7. Suppose that $\mathcal{N}(A) \cap \mathcal{X} \neq\{\mathbf{0}\}$. Consider 3 , and (9). If $f_{p c}^{*}(\mathbf{c})<f_{d c}^{*}$ where $\mathbf{c}=\operatorname{Proj}_{A \mathcal{X}}(\mathbf{b})$, the optimal value of (3) can be attainable.

Proof: See Appendix A

We are now in the stage to present the theorem on the existence of global solutions of (2) and (3). The proof follows the line of arguments as in [17, Theorem 3.4], thus omitted here.

Theorem 8. Consider (2) and (3). Suppose that $\mathcal{N}(A)$ has the $s$-spherical section property for some $s>0$. Then, one of the following assertions holds:

(i) There exists $\hat{\mathbf{x}} \in \mathbb{R}^{n}$ such that $\|\hat{\mathbf{x}}\|_{0}<m / s, \hat{\mathbf{x}} \in \mathcal{X}$ and $A \hat{\mathbf{x}}=\mathbf{b}$, the set of optimal solution of $(2)$ is nonempty. 
(ii) There exists $\hat{\mathbf{x}} \in \mathbb{R}^{n}$ such that $\|\hat{\mathbf{x}}\|_{0}<m / s$, $\hat{\mathbf{x}} \in \mathcal{X}$, and $A \hat{\mathbf{x}}=\mathbf{c}$ where $\mathbf{c}=\operatorname{Proj}_{A \mathcal{X}}(\mathbf{b})$, the set of optimal solutions of (3) is nonempty.

Next, we consider that how to guarantee $\mathbf{0}$ not being a global optimal solutions for (2) and (3). In view of $\mathbf{b} \neq \mathbf{0}, \mathbf{0}$ cannot be a global optimal solution of the constrained model (2). In Theorem 9 , we provide sufficient conditions to guarantee that 0 cannot be a global optimal solution of (3).

Theorem 9. Suppose that one of the following assumptions holds:

(i) $\mathbf{b} \in A \mathcal{X}$ and $0<\gamma<\frac{\|\mathbf{b}\|_{2}^{2}}{2(\sqrt{n}-1)}$,

(ii) There exists a vector $\hat{\mathbf{x}} \in \mathcal{X}$ such that $\|A \hat{\mathbf{x}}-\mathbf{b}\|_{2} \leq$ $\varepsilon\left(\varepsilon \ll\|\mathbf{b}\|_{2}\right)$ and $0<\gamma<\frac{\|\mathbf{b}\|_{2}^{2}-\varepsilon^{2}}{2(\sqrt{n}-1)}$.

Then, the optimal solution of (3) can not be $\mathbf{0}$.

Proof: (i) We use contradiction to show it. Suppose that 0 is a global solution of (3). Since $\mathbf{b} \in A \mathcal{X}$, we choose a vector $\tilde{\mathbf{x}} \in \mathcal{X}$ such that $A \tilde{\mathbf{x}}=\mathbf{b}$. Then, it leads to $\gamma \sqrt{n}<\gamma+\frac{1}{2}\|\mathbf{b}\|^{2}$ which implies that $F(\tilde{\mathbf{x}})<F(\mathbf{0})$. It contradicts to $\mathbf{0}$ being a global solution of (3). (ii) The proof is similar to (i), thus omitted here.

Remark 3. The assumptions of (i) and (ii) in Theorem 9 correspond to the cases of noiseless and noisy observations, respectively.

\section{COMPUTATIONAL APPROACH}

In this section, we focus on solving (3) with $\mathcal{X}=\mathbb{R}_{+}^{n}$. First, we derive the closed-form solution of $\left(L_{1} / L_{2}\right)^{+}$, and develop a practical solver for one of its global solution, which provides an extension of Moreau's proximal theory to nonconvex setting [29, 30].

\section{A. Proximal operator}

Define a proximal operator of $\left(L_{1} / L_{2}\right)^{+}$with a parameter $\rho>0$ as

$\operatorname{Prox}_{\left(L_{1} / L_{2}\right)^{+}}^{\rho}(\mathbf{q}):=\arg \min _{\mathbf{x} \geq \mathbf{0}}\left(\frac{\|\mathbf{x}\|_{1}}{\|\mathbf{x}\|_{2}}+\frac{\rho}{2}\|\mathbf{x}-\mathbf{q}\|_{2}^{2}\right)$.

Obviously, if $\mathbf{q} \leq 0$, the solution of $(11)$ is $\mathbf{0}$. For the rest of the paper, we assume $\mathbf{q} \not \leq 0$. Next, we construct one specific example, i.e., Example 3.1 to show that the optimal solution of (11) may not be unique.

Example 1. Let $n=2$ and $q_{1}=q_{2}=\sqrt{2(\sqrt{2}-1)}$. Consider an objective function

$$
\min _{\mathbf{x} \geq 0} \frac{\left|x_{1}\right|+\left|x_{2}\right|}{\sqrt{x_{1}^{2}+x_{2}^{2}}}+\frac{1}{2}\left(x_{1}-q_{1}\right)^{2}+\frac{1}{2}\left(x_{2}-q_{2}\right)^{2} .
$$

It has three global optimal solutions:

$$
\mathbf{x}_{1}=(\sqrt{2(\sqrt{2}-1)}, 0)^{\top}, \mathbf{x}_{2}=(0, \sqrt{2(\sqrt{2}-1)})^{\top}
$$

and $\mathbf{x}_{3}=(\sqrt{2(\sqrt{2}-1)}, \sqrt{2(\sqrt{2}-1)})^{\top}$.

In what follows, we characterize a closed-form solution of $\operatorname{Prox}_{\left(L_{1} / L_{2}\right)^{+}}^{\rho}(\mathbf{q})$ in Theorem 10 The assertion (i) of Theorem
10 presents the descent property of its entries for any optimal solution of (11), and the assertion (ii) aims to find one of the optimal solutions of $\operatorname{Prox}_{\left(L_{1} / L_{2}\right)^{+}}^{\rho}(\mathbf{q})$ in a closed form.

Theorem 10. Given $\mathbf{q} \in \mathbb{R}^{n}$ and $\mathbf{q} \not \leq \mathbf{0}$ and $\rho>0$. We can sort $\mathbf{q}$ in a descending order in a way of $q_{\pi(1)} \geq \cdots \geq$ $q_{\pi(\nu)}>0 \geq q_{\pi(\nu+1)} \geq \cdots \geq q_{\pi(n)} \geq 0$ where $\pi$ is a proper permutation of $[n]$. Suppose $\overline{\mathrm{x}}$ be an optimal solution of [11]. Then, the following assertions hold:

(i) For any optimal solution $\overline{\mathbf{x}}$ of (11), it has the same descending order as $\mathbf{q}$, i.e.,

$$
\bar{x}_{\pi(1)} \geq \cdots \geq \bar{x}_{\pi(\nu)} \geq 0=\bar{x}_{\pi(\nu+1)}=\cdots=\bar{x}_{\pi(n)} .
$$

(ii) We denote the multiplicity of the largest magnitude in $\mathbf{q}$ as $\mu$, i.e., $q_{\pi(1)}=\cdots=q_{\pi(\mu)}>q_{\pi(\mu+1)}$.

One of the following assertions holds:

(a) If $0<\rho \leq 1 /\left(q_{\pi(1)}^{2}\right)$, then (11) has a one-sparse solution given by

$$
\bar{x}_{\pi(i)}= \begin{cases}q_{\pi(i)} & i=1 \\ 0 & \text { otherwise. }\end{cases}
$$

(b) If $\rho>\frac{1}{q_{\pi(1)}^{2}}$, there exist an integer $t(t \leq \nu)$ and a scalar pair of $(a, r)$ such that $\left(Q^{t}=\sum_{i=1}^{t} q_{\pi(i)}\right)$

$$
\left\{\begin{array}{l}
\frac{a^{2}}{r^{3}}-\rho a+\rho Q^{t}-\frac{t}{r}=0 \\
r^{3}-\left(\sum_{i=1}^{t} q_{\pi(i)}^{2}\right) r+\frac{Q^{t}-a}{\rho}=0,
\end{array}\right.
$$

and the $r$ is also satisfied with

$$
q_{\pi(t)}-\frac{1}{\rho r}>0 \text { and } q_{\pi(t+1)}-\frac{1}{\rho r} \leq 0,
$$

and the vector $\overline{\mathrm{x}}$ is characterized by

$$
\bar{x}_{\pi(i)}= \begin{cases}\frac{\rho q_{\pi(i)}-\frac{1}{r}}{\rho-\frac{a}{r^{3}}} & 1 \leq i \leq t, \\ 0 & \text { otherwise },\end{cases}
$$

is an optimal solution of $(11)$, where $t=\|\overline{\mathbf{x}}\|_{0}$, $a=\|\overline{\mathbf{x}}\|_{1}, r=\|\overline{\mathbf{x}}\|_{2}$.

Proof: (i) First, since $q_{\pi(1)} \geq q_{\pi(2)} \geq \cdots \geq q_{\pi(n)}$, thus

$$
\bar{x}_{\pi(1)} \geq \bar{x}_{\pi(2)} \geq \cdots \geq \bar{x}_{\pi(n)} .
$$

If there exists several entries of $\mathbf{q}$ with the same value, the corresponding entries in $\overline{\mathbf{x}}$ can be arranged in a descending order. Therefore, we only need to verify that for $\bar{x}_{i}, \bar{x}_{j}>0$,

$$
q_{i}>q_{j} \Rightarrow \bar{x}_{i} \geq \bar{x}_{j} .
$$

We use contradiction. Define the objective function of (11) by $f(\mathbf{x}):=\frac{\|\mathbf{x}\|_{1}}{\|\mathbf{x}\|_{2}}+\iota_{\geq 0}(\mathbf{x})+\frac{\rho}{2}\|\mathbf{x}-\mathbf{q}\|_{2}^{2}$. Suppose not. It means that $\bar{x}_{i}<\bar{x}_{j}$. Then, we exchange these two entries of $\bar{x}_{i}$ and $\bar{x}_{j}$ in $\overline{\mathbf{x}}$, and denote the resultant vector as $\hat{\mathbf{x}}$. Then, with some elementary calculations, it follows that $f(\hat{\mathbf{x}})<f(\overline{\mathbf{x}})$ since

$$
\left(q_{i}-\bar{x}_{i}\right)^{2}+\left(q_{j}-\bar{x}_{j}\right)^{2}>\left(q_{i}-\bar{x}_{j}\right)^{2}+\left(q_{j}-\bar{x}_{i}\right)^{2} .
$$

Then, it contradicts to $\overline{\mathbf{x}}$ being a global optimal solution. It implies that 177 holds. Thus, 16 follows directly. 
Next, we show that

$$
q_{i} \leq 0 \Rightarrow \bar{x}_{i}=0 .
$$

We use contradiction to show it. Suppose that there exists at least one index $\hat{i}$ such that $q_{\hat{i}} \leq 0$ and $\bar{x}_{i}>0$. Since $\mathbf{q} \not \leq \mathbf{0}$, we have that $\overline{\mathbf{x}} \neq \mathbf{0}$. Suppose not, i.e., $\overline{\mathbf{x}}=\mathbf{0}$ and $\mathbf{q} \not \leq \mathbf{0}$. Then, there exists an index $i$ such that $q_{i}>0$. We introduce another vector $\mathbf{x}^{*} \in \mathbb{R}^{n}$, defined as $\mathbf{x}_{i}^{*}=q_{i}$ and $\mathbf{x}_{j}^{*}=0$ with $j \neq i$. It follows that $f\left(\mathbf{x}^{*}\right)<f(\overline{\mathbf{x}})$ which contradicts to $\overline{\mathbf{x}}$ being a global solution. Thus, $\overline{\mathbf{x}} \neq \mathbf{0}$.

Next, invoking the optimality of the conditions of (11), it leads to

$(\mathbf{x}-\overline{\mathbf{x}})^{\top}\left(\frac{\mathbf{1}}{\|\overline{\mathbf{x}}\|_{2}}-\frac{\|\overline{\mathbf{x}}\|_{1}}{\|\mathbf{x}\|_{2}^{3}} \overline{\mathbf{x}}+\rho(\overline{\mathbf{x}}-\mathbf{q})\right) \geq \mathbf{0}, \quad \forall \mathbf{x} \geq \mathbf{0}$.

Define $\Upsilon:=\frac{\mathbf{1}}{\|\overline{\mathbf{x}}\|_{2}}-\frac{\|\overline{\mathbf{x}}\|_{1}}{\|\overline{\mathbf{x}}\|_{2}^{3}} \overline{\mathbf{x}}+\rho(\overline{\mathbf{x}}-\mathbf{q})$. Then, it leads to

$$
\bar{x}_{i}>0 \Rightarrow \Upsilon_{i}=0 .
$$

In the following, we divide into two cases to verify. Denote $a=\|\overline{\mathbf{x}}\|_{1}$ and $r=\|\overline{\mathbf{x}}\|_{2}$. Case $1 . \rho \geq a / r^{3}$. Since $\bar{x}_{i}>0$, $\Upsilon_{i}=\frac{1}{\|\overline{\mathbf{x}}\|_{2}}-\frac{a}{r^{3}} \bar{x}_{i}+\rho\left(\bar{x}_{i}-q_{i}\right)>0$ which contradicts to 19 . Case 2. $\rho<a / r^{3}$. Invoking (18), it leads to

$$
\bar{x}_{i}=\frac{\rho q_{i}-\frac{1}{r}}{\rho-\frac{a}{r^{3}}}, \quad \forall i \in \operatorname{supp}(\overline{\mathbf{x}}) .
$$

We define two index sets: $\Lambda^{+}:=\left\{i \mid q_{i}>0\right\}$ and $\Lambda^{-}:=$ $\left\{i \mid q_{i} \leq 0\right\}$. We divide $\overline{\mathbf{x}}$ into two parts: $\overline{\mathbf{x}}^{+}=\left.\overline{\mathbf{x}}\right|_{\Lambda^{+}}$and $\overline{\mathbf{x}}^{-}=\left.\overline{\mathbf{x}}\right|_{\Lambda^{-}}$. By assumption, we known that $\overline{\mathbf{x}}^{-} \neq \mathbf{0}$. Thus, $\overline{\mathbf{x}}^{+} \neq \mathbf{0}$ due to 16 and 17 . Picking up $i \in \Lambda^{+}$and setting $j=\hat{i}\left(\in \Lambda^{-}\right)$, it leads to $q_{i}>q_{j}$. Consequently,

$$
\bar{x}_{i}<\bar{x}_{j},
$$

due to $\rho<a / r^{3}$. On the other hand, since $\overline{\mathbf{x}}$ is an optimal solution, we have proved that

$$
q_{i}>q_{j} \Rightarrow \bar{x}_{i} \geq \bar{x}_{j},
$$

which contradicts to $(20)$. Therefore, the assertion $(18)$ holds. Thus, the assertion (i) follows immediately. (ii) In view of (18), it implies that the minimization problem (11) amounts to solving a low-dimension minimization problem:

$$
\arg \min _{\mathbf{y} \in \mathbb{R}^{\nu}}\left(\frac{\|\mathbf{y}\|_{1}}{\|\mathbf{y}\|_{2}}+\frac{\rho}{2}\|\mathbf{y}-\mathbf{p}\|_{2}^{2}\right),
$$

where $\mathbf{p}=\left.\mathbf{q}\right|_{\sigma}$ and $\sigma:=\{\pi(1), \cdots, \pi(\nu)\}$. Denoting $\overline{\mathbf{y}}$ as an optimal solution of 21, the vector $\mathrm{x}$ defined by $\left.\mathbf{x}\right|_{\sigma}=\overline{\mathbf{y}}$ and $\left.\mathbf{x}\right|_{\sigma^{c}}=\mathbf{0}$ is an optimal solution (11). By invoking [15, Theorem 3.3], the assertion of (ii) follow immediately.

From the proof to Theorem 10 and following up the discussions in [15, Section 3], we can use [15, Algorithm 3.1] to solve the dimension-reduced problem (21). More details can be found in [15]. We summarize the practical version for (11) in Algorithm 11 where $\operatorname{Prox}_{\left(L_{1} / L_{2}\right)}^{\rho}(\mathbf{p}):=\arg \min _{\mathbf{y}}\left(\|\mathbf{\| y}\|_{1}\right.$ $\left.\frac{\rho}{2}\|\mathbf{y}-\mathbf{p}\|_{2}^{2}\right)$.

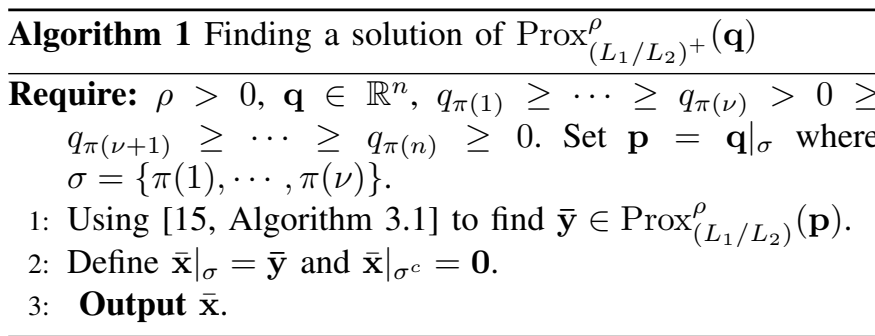

\section{B. ADMM for solving (3) with $\mathcal{X}=\mathbb{R}_{+}^{n}$}

Although there exist a few different ways for reformulating the unconstrained model 3 with $\mathcal{X}=\mathbb{R}_{+}^{n}$, most of them result in a scheme of ADMM with violation of convergence guarantee. Equipped with the newly-developed solution of the proximity of $\left(L_{1} / L_{2}\right)^{+}$, we apply ADMM to its equivalence reformulation of $(3)$, i.e.,

$$
\begin{array}{ll}
\min _{\mathbf{x}, \mathbf{y} \in \mathcal{X}} & \gamma \frac{\|\mathbf{x}\|_{1}}{\|\mathbf{x}\|_{2}}+\frac{1}{2}\|A \mathbf{y}-\mathbf{b}\|_{2}^{2} \\
\text { s.t. } & \mathbf{x}=\mathbf{y}, \mathbf{x} \in \mathcal{X} .
\end{array}
$$

We define the augmented Lagrangian of 22 as

$$
\begin{array}{r}
\mathcal{L}_{\mathcal{A}}(\mathbf{x}, \mathbf{y}, \mathbf{z})=\gamma \\
\frac{\|\mathbf{x}\|_{1}}{\|\mathbf{x}\|_{2}}+\iota \mathcal{X}(\mathbf{x})+\frac{1}{2}\|A \mathbf{y}-\mathbf{b}\|^{2} \\
+\mathbf{z}^{\top}(\mathbf{x}-\mathbf{y})+\frac{\beta}{2}\|\mathbf{x}-\mathbf{y}\|_{2}^{2},
\end{array}
$$

where $\mathbf{z}$ is the Lagrangian multiplier and $\beta>0$ is the penalty parameter. Given $\left(\mathbf{y}^{k}, \mathbf{z}^{k}\right)$, the ADMM scheme generates the iterative sequence $\left\{\mathbf{w}^{k}\right\}\left(\mathbf{w}^{k}=\left(\mathbf{x}^{k}, \mathbf{y}^{k}, \mathbf{z}^{k}\right)\right)$ as follows,

$$
\left\{\begin{array}{l}
\mathbf{x}^{k+1} \in \arg \min _{\mathbf{x} \in \mathcal{X}} \mathcal{L}_{\mathcal{A}}\left(\mathbf{x}, \mathbf{y}^{k}, \mathbf{z}^{k}\right), \\
\mathbf{y}^{k+1}=\arg \min _{\mathbf{y}} \mathcal{L}_{\mathcal{A}}\left(\mathbf{x}^{k+1}, \mathbf{y}, \mathbf{z}^{k}\right), \\
\mathbf{z}^{k+1}=\mathbf{z}^{k}+\beta\left(\mathbf{x}^{k+1}-\mathbf{y}^{k+1}\right) .
\end{array}\right.
$$

The $\mathrm{x}$-subproblem 23a amounts to

$$
\mathbf{x}^{k+1} \in \arg \min _{\mathbf{x} \in \mathcal{X}}\left(\gamma \frac{\|\mathbf{x}\|_{1}}{\|\mathbf{x}\|_{2}}+\frac{\beta}{2}\left\|\mathbf{x}-\mathbf{y}^{k}+\frac{\mathbf{z}^{k}}{\beta}\right\|_{2}^{2}\right),
$$

which coincides with $\operatorname{Prox}_{\left(L_{1} / L_{2}\right)^{+}}^{\rho}(\mathbf{q})$ with $\mathbf{q}:=\mathbf{y}^{k}-\mathbf{z}^{k} / \beta$ and $\rho:=\beta / \gamma$. It deserves a closed-form solution (i.e., Theorem 10]. The $\mathbf{y}$-subproblem 23b is given by

$$
\mathbf{y}^{k+1}=\left(I_{n}+\frac{1}{\beta} A^{\top} A\right)^{-1}\left(\frac{A^{\top} \mathbf{b}}{\beta}+\frac{\mathbf{z}^{k}}{\beta}+\mathbf{x}^{k+1}\right) .
$$

By using Sherman-Morrison-Woodburg Theorem, it leads to a more efficient scheme:

$$
\mathbf{y}^{k+1}=M\left(\frac{A^{\top} \mathbf{b}}{\beta}+\frac{\mathbf{z}^{k}}{\beta}+\mathbf{x}^{k+1}\right),
$$

where $M=I_{n}-\frac{1}{\beta} A^{\top}\left(I_{m}+\frac{1}{\beta} A A^{\top}\right)^{-1} A$ because $m \ll n$. We summarize the overall implementing scheme of 23) for the model (3) with $\mathcal{X}=\mathbb{R}_{+}^{n}$ in Algorithm 2 and referred to as $\mathrm{ADMM}_{p}^{+}$. 


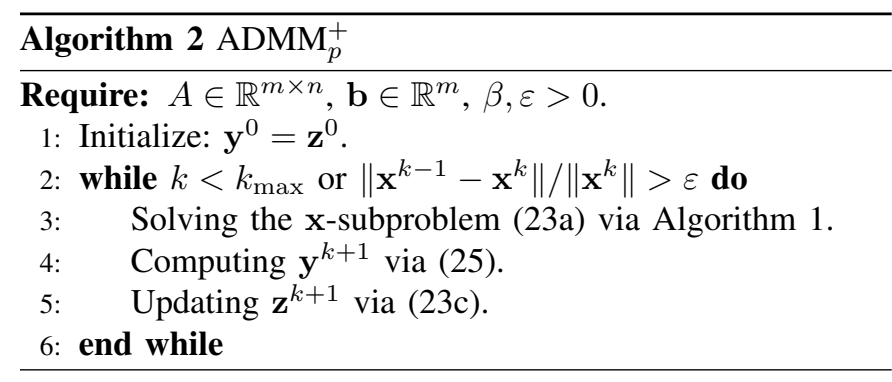

\section{Global Convergence}

In order to establish the global convergence, we introduce the following potential function $\left(\mathcal{X}:=\mathbb{R}_{+}^{n}\right)$,

$\mathcal{T}(\mathbf{x}, \mathbf{y})=\gamma \frac{\|\mathbf{x}\|_{1}}{\|\mathbf{x}\|_{2}}+\iota \mathcal{X}(\mathbf{x})+\frac{1}{2}\|A \mathbf{x}-\mathbf{b}\|^{2}+\frac{\beta}{2}\|\mathbf{x}-\mathbf{y}\|_{2}^{2}$

Similar to [15, Lemma 5.6], we show the sufficient decay of the function $\mathcal{T}$ in Lemma 5 . Then we provide an upper bound for the distance between the subgradient of $T$ and the zero vector in terms of residual between two adjacent iterates in Lemma 6. In the following, we denote $\mathcal{T}^{k}:=\mathcal{T}\left(\mathbf{x}^{k}, \mathbf{y}^{k}\right)$ for succinctness.

Lemma 5. Let $\left\{\mathrm{w}^{k}\right\}$ be the sequence generated by $(23)$ and $\mathcal{T}(\mathbf{x}, \mathbf{y})$ defined in $(26)$. If $\beta>2 L$, then there exists a constant $c_{1}>0$ such that

$$
\mathcal{T}\left(\mathbf{x}^{k+1}, \mathbf{y}^{k+1}\right) \leq \mathcal{T}\left(\mathbf{x}^{k}, \mathbf{y}^{k}\right)-c_{1}\left\|\mathbf{y}^{k}-\mathbf{y}^{k+1}\right\|_{2}^{2} .
$$

Proof: First, it follows from the optimality condition of 23b that $\mathbf{z}^{k+1}=A^{\top}\left(A \mathbf{y}^{k+1}-\mathbf{b}\right)$. Then, it further implies that

$$
\left\|\mathbf{z}^{k}-\mathbf{z}^{k+1}\right\|_{2} \leq L\left\|\mathbf{y}^{k}-\mathbf{y}^{k+1}\right\|_{2},
$$

where $L=\sigma_{\max }\left(A^{\top} A\right)$ where $\sigma_{\max }(\cdot)$ represents the largest eigenvalue. Next, invoking 23a, it leads to $\mathcal{L}_{\mathcal{A}}\left(\mathbf{x}^{k+1}, \mathbf{y}^{k}, \mathbf{z}^{k}\right) \leq \mathcal{L}_{\mathcal{A}}\left(\mathbf{x}^{k}, \mathbf{y}^{k}, \mathbf{z}^{k}\right)$. Then, using 23b, it yields that $\mathcal{L}_{\mathcal{A}}\left(\mathbf{x}^{k+1}, \mathbf{y}^{k+1}, \mathbf{z}^{k}\right) \leq \mathcal{L}_{\mathcal{A}}\left(\mathbf{x}^{k+1}, \mathbf{y}^{k}, \mathbf{z}^{k}\right)-$ $\frac{\beta}{2}\left\|\mathbf{y}^{k}-\mathbf{y}^{k+1}\right\|^{2}$. In view of $\left.\sqrt{23 \mathrm{c}}\right)$, we obtain that $\mathcal{L}_{\mathcal{A}}\left(\mathbf{x}^{k+1}, \mathbf{y}^{k+1}, \mathbf{z}^{k+1}\right)=\mathcal{L}_{\mathcal{A}}\left(\mathbf{x}^{k+1}, \mathbf{y}^{k+1}, \mathbf{z}^{k}\right)+\frac{1}{\beta} \| \mathbf{z}^{k}-$ $\mathbf{z}^{k+1} \|^{2}$. Combining above three inequalities with 23c, we have that

$$
\begin{aligned}
& \mathcal{L}_{\mathcal{A}}\left(\mathbf{x}^{k+1}, \mathbf{y}^{k+1}, \mathbf{z}^{k+1}\right) \\
& \leq \mathcal{L}_{\mathcal{A}}\left(\mathbf{x}^{k}, \mathbf{y}^{k}, \mathbf{z}^{k}\right)-c_{1}\left\|\mathbf{y}^{k}-\mathbf{y}^{k+1}\right\|^{2},
\end{aligned}
$$

where $c_{1}=\beta / 2-L^{2} / \beta$. Consequently, Consequently,

$$
\begin{aligned}
\mathcal{T}^{k+1} & \leq \mathcal{L}_{\mathcal{A}}\left(\mathbf{x}^{k+1}, \mathbf{y}^{k+1}, \mathbf{z}^{k+1}\right)+\frac{L}{2}\left\|\mathbf{x}^{k+1}-\mathbf{y}^{k+1}\right\|^{2} \\
& \leq \mathcal{L}_{\mathcal{A}}\left(\mathbf{x}^{k}, \mathbf{y}^{k}, \mathbf{z}^{k}\right)-\left(\beta / 2-L^{2} / \beta\right)\left\|\mathbf{y}^{k}-\mathbf{y}^{k+1}\right\|^{2} \\
& +\frac{L}{2 \beta^{2}}\left\|\mathbf{z}^{k}-\mathbf{z}^{k+1}\right\|^{2} \\
& \leq \mathcal{L}_{\mathcal{A}}\left(\mathbf{x}^{k}, \mathbf{y}^{k}, \mathbf{z}^{k}\right)-\frac{3 L}{8}\left\|\mathbf{y}^{k}-\mathbf{y}^{k+1}\right\|^{2},
\end{aligned}
$$

where the first inequality is due to $\mathbf{z}^{k+1}=A^{\top}\left(A \mathbf{y}^{k+1}-\mathbf{b}\right)$ and Lipchitz continuous of the function $A^{\top}(A(\cdot)-\mathbf{b})$, the second is due to (23c) and (29), and the last is due to 28 and $\beta>2 L$. On the other hand, since $\mathbf{z}^{k}=A^{\top}\left(A \mathbf{y}^{k}-\mathbf{b}\right)$, it leads to $\mathcal{T}^{k} \geq \mathcal{L}_{\mathcal{A}}\left(\mathbf{x}^{k}, \mathbf{y}^{k}, \mathbf{z}^{k}\right)$. Combining these two inequalities, the assertion follows directly.

The proof of the following lemma is similar to [15, Lemma 5.7] and thus omitted.

Lemma 6. Let $\left\{\mathbf{w}^{k}\right\}$ be the sequence generated by 23 . Then there exists a constant $c_{2}>0$ such that

$$
\operatorname{dist}\left(\mathbf{0}, \partial \mathcal{T}\left(\mathbf{x}^{k+1}, \mathbf{y}^{k+1}\right)\right) \leq c_{2}\left\|\mathbf{y}^{k+1}-\mathbf{y}^{k}\right\|_{2} .
$$

Next, we prove that the subsequential convergence of 23 to a d-stationary point of (3).

Theorem 11. Let $\left\{\mathrm{w}^{k}\right\}$ be the sequence generated by (23). If $\left\{\mathrm{x}^{k}\right\}$ is bounded and $\beta>2 L$, we have the following statements:

(i) $\lim _{k \rightarrow \infty}\left\|\mathbf{x}^{k}-\mathbf{x}^{k+1}\right\|=0, \lim _{k \rightarrow \infty}\left\|\mathbf{y}^{k}-\mathbf{y}^{k+1}\right\|=0$, and $\lim _{k \rightarrow \infty}\left\|\mathbf{z}^{k}-\mathbf{z}^{k+1}\right\|=0$;

(ii) The sequence $\left\{\mathbf{w}^{k}\right\}$ has at least one accumulation point;

(iii) Any accumulation point of $\left\{\mathrm{x}^{k}\right\}$ is a d-stationary point of (22).

Proof: (i) In view of (27), $\lim _{k \rightarrow \infty}\left\|\mathbf{y}^{k}-\mathbf{y}^{k+1}\right\|=$ 0 is ensured immediately. Furthermore, (28) leads to $\lim _{k \rightarrow \infty}\left\|\mathbf{z}^{k}-\mathbf{z}^{k+1}\right\|=0$. Obviously, $\mathbf{x}^{k+1}-\mathbf{x}^{k}=\frac{1}{\beta}\left(\left(\mathbf{z}^{k+1}-\right.\right.$ $\left.\left.\mathbf{z}^{k}\right)-\left(\mathbf{z}^{k}-\mathbf{z}^{k-1}\right)\right)+\left(\mathbf{y}^{k+1}-\mathbf{y}^{k}\right)$. Thus, $\lim _{k \rightarrow \infty} \| \mathbf{x}^{k}-$ $\mathbf{x}^{k+1} \|=0$.

(ii) Since $\left\{\mathbf{x}^{k}\right\}$ is bounded and $\left\|\mathbf{x}^{k}-\mathbf{y}^{k}\right\|_{2} \rightarrow 0$, it leads to the boundedness of $\left\{\mathbf{y}^{k}\right\}$. Furthermore, in view of $\mathbf{z}^{k}=$ $A^{\top}\left(A \mathbf{y}^{k}-\mathbf{b}\right)$, it implies that $\left\{\mathbf{z}^{k}\right\}$ is bounded. Therefore, the sequence of $\left\{\mathbf{x}^{k}, \mathbf{y}^{k}, \mathbf{z}^{k}\right\}$ is bounded and hence it has at least one accumulation point, i.e., $\mathbf{w}^{\infty}$. It is satisfied with

$\left\{\begin{array}{l}\left(\mathbf{x}-\mathbf{x}^{\infty}\right)^{\top}\left(\frac{\mathbf{p}^{\infty}}{\left\|\mathbf{x}^{\infty}\right\|_{2}}-\frac{\left\|\mathbf{x}^{\infty}\right\|_{1}}{\left\|\mathbf{x}^{\infty}\right\|_{2}^{3}} \mathbf{x}^{\infty}+\mathbf{z}^{\infty}\right) \geq 0 \forall \mathbf{x} \in \mathcal{X} \\ A^{\top}\left(A \mathbf{y}^{\infty}-\mathbf{b}\right)-\mathbf{z}^{\infty}=0 \\ \mathbf{x}^{\infty}=\mathbf{y}^{\infty}\end{array}\right.$

where $\mathbf{p}^{\infty} \in \operatorname{sign}\left(\mathbf{x}^{\infty}\right)$. (iii) Suppose that $\left(\mathbf{x}^{*}, \mathbf{y}^{*}\right)$ be an accumulation point of $\left\{\mathbf{x}^{k}, \mathbf{y}^{k}\right\}$. Then, there exists an vector $\mathbf{z}^{*}$ such that $\left(\mathbf{x}^{*}, \mathbf{y}^{*}, \mathbf{z}^{*}\right)$ is an accumulation point of $\left(\mathbf{x}^{k}, \mathbf{y}^{k}, \mathbf{z}^{k}\right)$ and satisfied with 30 . It further implies that $\mathrm{x}^{*}$ is satisfied with (5) by taking $\overline{\mathbf{x}}=\mathbf{x}^{*}$. The conclusion follows directly.

We are now ready to establish the global convergence of $\mathrm{ADMM}_{p}^{+}$to a d-stationary point in Theorem 12

Theorem 12. Let $\left\{\mathbf{w}^{k}\right\}$ be the sequence generated by $(23)$. If $A^{\top} \mathbf{b} \not \leq \mathbf{0}, \beta>2 L$, and $\left\{\mathbf{x}^{k}\right\}$ is bounded, then $\left\{\mathbf{w}^{k}\right\}$ has finite length, i.e., $\sum_{k=1}^{\infty}\left\|\mathbf{w}^{k+1}-\mathbf{w}^{k}\right\|<\infty$, and hence $\left\{\mathbf{w}^{k}\right\}$ converges to a stationary point $\mathbf{w}^{\infty}:=\left(\mathbf{x}^{\infty}, \mathbf{y}^{\infty}, \mathbf{z}^{\infty}\right)$ in the sense of (30) where $\mathbf{x}^{\infty}$ is a d-stationary point of (3).

Proof: According to [31], if at least one of the two subanalytic functions maps bounded sets to bounded sets, then their sum is subanalytic. Since $\frac{1}{2}\|A \mathbf{x}-\mathbf{b}\|_{2}^{2}$ is real analytic and maps bounded sets to bounded sets, and the function $\gamma \frac{\|\mathbf{x}\|_{1}}{\|\mathbf{x}\|_{2}}+\iota_{\mathbb{R}_{+}^{n}}(\mathbf{x})$ is subanalytic, then their sum is also subanalytic. Similarly, the function $\mathcal{T}(\mathbf{x}, \mathbf{y})$ defined in 26) is also subanalytic. Next, we show that any accumulation point $\mathbf{x}^{\infty}$ of the sequence $\left\{\mathrm{x}^{k}\right\}$ generated by 23 cannot be 
0. Suppose not. Then, there exists a subsequence of $\left\{\mathbf{w}^{k_{j}}\right\}$ converging to $\mathbf{w}^{\infty}$ satisfied with 30 where $\mathrm{x}^{k_{j}} \rightarrow \mathrm{x}^{\infty}=\mathbf{0}$. Thus, $\mathbf{x}^{k_{j}+1} \rightarrow \mathbf{x}^{\infty}=\mathbf{0}$. In view of 23a and Theorem 10 . one has $\mathbf{y}^{k_{j}}-\frac{1}{\beta} \mathbf{z}^{k_{j}} \rightarrow \boldsymbol{\xi}^{\infty}:=\mathbf{y}^{\infty}-\frac{1}{\beta} \mathbf{z}^{\infty} \leq \mathbf{0}$. On the other hand, $\mathbf{y}^{k_{j}} \rightarrow \mathbf{y}^{\infty}=\mathbf{0}$ due to $\mathbf{x}^{\infty}-\mathbf{y}^{\infty}=\mathbf{0}$. Thus, $\mathbf{z}^{k_{j}} \rightarrow-\beta \boldsymbol{\xi}^{\infty} \geq \mathbf{0}$. Invoking $\mathbf{z}^{k_{j}}=A^{\top}\left(A \mathbf{y}^{k_{j}}-\mathbf{b}\right)$ and letting $j \rightarrow \infty$, it leads to $A^{\top} \mathbf{b}=\beta \boldsymbol{\xi}^{\infty}$ which contradicts to $A^{\top} \mathbf{b} \not \leq \mathbf{0}$. Thus, $\mathbf{x}^{\infty} \neq \mathbf{0}$.

Furthermore, invoking Theorem 11 and Lemma 6, any accumulation point $\left(\mathbf{x}^{\infty}, \mathbf{y}^{\infty}\right)$ of $\left\{\mathbf{x}^{k}, \mathbf{y}^{k}\right\}$ generated from 23 is satisfied with $\mathbf{0} \in\left(\partial_{\mathbf{x}} \mathcal{T}\left(\mathbf{x}^{\infty}, \mathbf{y}^{\infty}\right), \partial_{\mathbf{y}} \mathcal{T}\left(\mathbf{x}^{\infty}, \mathbf{y}^{\infty}\right)\right)$, and $\mathbf{x}^{\infty}=\mathbf{y}^{\infty}$. Thus, $\left(\mathbf{x}^{\infty}, \mathbf{y}^{\infty}\right)$ can not be $(\mathbf{0}, \mathbf{0})$. Define $\tilde{\mathcal{U}}=$ $\left\{\mathbf{u}:=(\mathbf{x}, \mathbf{y}) \in \mathbb{R}^{n} \times \mathbb{R}^{n} \mid\|\mathbf{u}\|_{2} \geq \epsilon\right\}$ with $0<\epsilon<\frac{1}{2}\left\|\mathbf{u}^{\infty}\right\|_{2}$. In view of [26, Theorem 3.1], the potential function $\left.\mathcal{T}(\mathbf{x}, \mathbf{y})\right|_{\tilde{\mathcal{U}}}$ satisfies the KL property since $\left.\mathcal{T}(\mathbf{x}, \mathbf{y})\right|_{\tilde{\mathcal{U}}}$ is continuous and its domain is closed. Therefore, the function $\mathcal{T}(\mathbf{x}, \mathbf{y})$ satisfies the KL property at the stationary point $\left(\mathrm{x}^{\infty}, \mathrm{x}^{\infty}\right)$. The remaining proof is standard and similar to [18, Theorem 4], thus omitted here.

\section{Linear Convergence}

In this subsection, we prove its local linear convergence rate of (23) under suitable conditions.

Theorem 13. Let $\left\{\mathbf{w}^{k}\right\}$ be the sequence generated by 23 . If $A^{\top} b \not \mathbf{0}, \beta>2 L$, and $\left\{\mathbf{x}^{k}\right\}$ is bounded, then $\left\{\mathbf{w}^{k}\right\}$ converges to a stationary point $\mathrm{w}^{\infty}=\left(\mathrm{x}^{\infty}, \mathbf{y}^{\infty}, \mathbf{z}^{\infty}\right)$ where $\mathrm{x}^{\infty}$ is a d-stationary point of $(3)$. Let $\bar{\Lambda}:=\operatorname{supp}\left(\mathrm{x}^{\infty}\right)$. Suppose that $A_{\bar{\Lambda}}^{\top} A_{\bar{\Lambda}} \succ 0$. Then, there exists a scalar $\bar{\gamma}>0$ such that the sequence of $\left\{\mathbf{w}^{k}\right\}$ converges linearly when $0<\gamma<\bar{\gamma}$, i.e., there exist $c_{1}>0$ and $\nu \in[0,1)$ such that

$$
\left\|\mathbf{w}^{k}-\mathbf{w}^{\infty}\right\|_{2} \leq c_{1} \nu^{k} .
$$

Proof: See Appendix B

\section{NUMERICAL RESULTS}

In this section, we examine two specific applications where the sensing matrix is highly coherent, i.e., recovery of nonnegative signal from (a) coherent dictionaries, and (b) wavelength misalignment in different optical absorption spectroscopy (DOAS) analysis, respectively.

All these algorithms are implemented on MATLAB R2016a and the experiments are performed on a desktop with Windows 10 and an Intel Core i7-7600U CPU processor $(2.80 \mathrm{GH})$ with $16 \mathrm{~GB}$ memory. The stopping criterion is set to meet one of the conditions:

RelChg $:=\frac{\left\|\mathbf{x}^{k}-\mathbf{x}^{k-1}\right\|_{2}}{\max \left\{\left\|\mathbf{x}^{k-1}\right\|_{2}, 0.1\right\}}<$ Tol or $k_{\max }>10 n$.

We set Tol as

$$
\mathrm{Tol}= \begin{cases}10^{-4} & \text { if } \sigma=0, \\ 0.01 * \sigma & \text { if } \sigma>0,\end{cases}
$$

where $\sigma$ is the variance of the noise $(\sigma=0$ means the noiseless case).

\section{A. Recovery nonnegative signal from coherent dictionaries}

In this subsection, we illustrate the efficiency of $\mathrm{ADMM}_{p}^{+}$ by solving Examples 1-3 of [8] via (3) with $\mathcal{X}=\mathbb{R}_{+}^{n}$. These examples are constructed to show the superiority of $L_{1} / L_{2}$ minimization over $L_{1}$ or $L_{p}(0<p<1)$ (i.e., $\left.\|\cdot\|_{p}\right)$ minimization.

Example 1: Let $p \in(0,1]$ and two distinct dense vectors $\mathbf{b}^{1}, \mathbf{b}^{2} \in \mathbb{R}^{n}\left(n \geq 2, \mathbf{b}^{2} \neq \mathbf{b}^{1}\right)$. Both of them are dense vectors and $\frac{\left\|\mathbf{b}^{i}\right\|_{1}}{\left\|\mathbf{b}^{i}\right\|_{2}}$ be close to $\sqrt{n}(i=1,2) . a=$ $\left\|\left(\mathbf{b}^{1}, \mathbf{b}^{2}\right)\right\|_{p}, A^{(1)}=\left[\mathbf{b}^{1}, \mathbf{b}^{2}, a I_{n}, a I_{n}\right]$. Consider the linear system $A^{(1)} \mathbf{x}=\mathbf{b}^{(1)}$ where $\mathbf{b}^{(1)}:=\mathbf{b}^{1}+\mathbf{b}^{2}$. Obviously, it has a two-sparse solution:

$$
\mathbf{x}^{(1)}=[1,1,0, \cdots, 0]^{\top} .
$$

Example 2: Let $p \in(0,1]$ and a dense vector $\mathbf{b} \in \mathbb{R}^{n}(n \geq$ 2), $a=\|(\mathbf{b}, \mathbf{b})\|_{p}=2^{1 / p}\|\mathbf{b}\|_{p}, A^{(2)}=\left[\mathbf{b}, \mathbf{b}, a I_{n}, a I_{n}\right]$ and $\mathbf{b}^{(2)}=2 \mathbf{b}$. The linear system $A^{(2)} \mathbf{x}=\mathbf{b}^{(2)}$ has a one-sparse solution:

$$
\mathbf{x}^{(2)}=[2,0, \cdots, 0]^{\top} .
$$

Example 3: Let $A^{(3)}:=\left[\mathbf{b}^{1}, \mathbf{b}^{2}, I_{n}, a I_{n}\right]$ where $\mathbf{b}^{1}, \mathbf{b}^{2}$ are the same as Example 1 and $\mathbf{b}^{(3)}=\mathbf{b}^{1}+\mathbf{e}^{1}$, where $\mathbf{e}^{1}=$ $[1,0, \cdots 0]^{\top}, a=2^{1 / p}\left\|\mathbf{b}^{1}\right\|_{p}(p \in(0,1]), \mathbf{b}^{2} \neq \mathbf{b}^{1}$, both are dense.

First, by setting $(A, \mathbf{b})=\left(\frac{A^{(i)}}{\left\|A^{(i)}\right\|_{2}}, \frac{\mathbf{b}^{(i)}}{\left\|A^{(i)}\right\|_{2}}\right)$ with $i=$ $1,2,3$ in 3 , respectively. We compare $\mathrm{ADMM}_{p}^{+}$with the scaled gradient projection method (SGPM) proposed in [13] and also used in [8]. We test on Examples 1-3, all these matrices $A^{(i)}(i=1,2,3)$ defined with values of $n=50,100$ and $p=0.9,0.95$, and the vectors of $\mathbf{b}^{(i)}(i=1,2,3)$ are of $n$ random numbers subjected to uniform distribution on $[0,1]$. The model parameter of (3) is $\gamma=0.01$ for Examples 1-3. For the SGPM, we use the defaulted setting as in [8, 13], i.e., $\delta=1, c_{0}=10^{-9}, \xi_{1}=2, \xi_{2}=10$ and $\sigma=0.01$. Set $\beta=0.8$ in $\mathrm{ADMM}_{p}^{+}$. For these three examples, we set the initial point as $x^{0}=0.05\left(100+0.01 \eta_{i}\right)$ and $\eta_{i} \sim N(0,1)$. In order to measure the extent of satisfying optimality condition of (5), we define the Karush-Kuhn-Tucker (KKT) residual on the support set $\left(\mathrm{KKT}_{R}\right)$ of the last iterate $\hat{\mathrm{x}}$ as:

$$
\operatorname{KKT}_{R}=\left\|\gamma\left(\frac{\operatorname{sign}\left(\hat{\mathbf{x}}_{\hat{\Lambda}}\right)}{\|\hat{\mathbf{x}}\|_{2}}-\frac{\|\hat{\mathbf{x}}\|_{1}}{\|\hat{\mathbf{x}}\|_{2}^{3}} \hat{\mathbf{x}}_{\hat{\Lambda}}\right)+\left(A_{\hat{\Lambda}}\right)^{\top}(A \hat{\mathbf{x}}-\mathbf{b})\right\|_{2},
$$

where $\hat{\Lambda}=\operatorname{supp}(\hat{\mathrm{x}})$.

In Table I. we record the results of $\mathrm{ADMM}_{p}^{+}(\mathrm{AD})$ and SGPM (SG) in terms of final objective function value (Obj.) of (3), the $\mathrm{KKT}_{R}$ and computational time (Time (s)). Table I clearly shows that $\mathrm{ADMM}_{p}^{+}$performs much better than SGPM in terms of achieving much lower objective function values, ending up with a much more accurate solution while taking less time. For the scenario of $n=50$ and $p=0.9$ of Example 2 , we find that $\mathrm{ADMM}_{p}^{+}$recovers the one-sparse solution $\mathbf{x}^{(2)}=[2,0, \cdots, 0]^{\top}$ while SGPM does not. 
TABLE I

COMPARISONS BETWEEN ADMM AM $^{+}$(AD) AND SGPM (SG) ON EXAMPLES 1, 2 AND 3 VIA SOLVING 3 . ADMM $_{p}^{+}$PERFORMS MUCH BETTER THAN SGPM IN TERMS OF ACHIEVING MUCH LOWER OBJECTIVE FUNCTION VALUES OF (3) WITH HIGHER ACCURACY.

\begin{tabular}{|c|c|c|c|c|}
\hline \multirow[t]{2}{*}{$($ Ex. $, n, p)$} & Obj. of 3 & \multirow{2}{*}{\multicolumn{2}{|c|}{$\begin{array}{c}\text { KKT }_{R} \\
\text { SG|AD }\end{array}$}} & Time (s) \\
\hline & $\mathrm{SG} \mid \mathrm{AD}$ & & & SG $\mid \mathrm{AD}$ \\
\hline$(1,50,0.95)$ & $5.64 \mid \mathbf{0 . 0 1 1}$ & 3.35 & $2.18 \times 10^{-4}$ & $0.63 \mid \mathbf{0 . 3 3}$ \\
\hline$(1,50,0.9)$ & $5.63 \mid \mathbf{0 . 0 1 0}$ & 3.35 & $4.38 \times 10^{-4}$ & $0.64 \mid \mathbf{0 . 2 5}$ \\
\hline$(1,100,0.95)$ & $11.2 \mid \mathbf{0 . 0 1 0}$ & 4.73 & $1.22 \times 10^{-4}$ & $2.11 \mid \mathbf{0 . 9 7}$ \\
\hline$(1,100,0.9)$ & $11.2 \mid \mathbf{0 . 0 1 0}$ & 4.73 & $2.69 \times 10^{-4}$ & $2.02 \mid \mathbf{0 . 8 1}$ \\
\hline$(2,50,0.95)$ & $\begin{array}{l}5.64 \mid \mathbf{0 . 0 1 0} \\
\end{array}$ & 3.35 & $5.69 \times 10^{-6}$ & $\begin{array}{l}0.61 \mid \mathbf{0 . 4 5} \\
\end{array}$ \\
\hline$(2,50,0.9)$ & $5.63 \mid \mathbf{0 . 0 1 0}$ & 3.35 & $1.05 \times 10^{-5}$ & $0.59 \mid \mathbf{0 . 1 6}$ \\
\hline$(2,100,0.95)$ & $11.2 \mid \mathbf{0 . 0 1 0}$ & 4.73 & $9.92 \times 10^{-6}$ & $1.72 \mid \mathbf{0 . 9 2}$ \\
\hline$(2,100,0.9)$ & $11.2 \mid \mathbf{0 . 0 1 0}$ & 4.72 & $1.18 \times 10^{-5}$ & $1.98 \mid \mathbf{0 . 2 7}$ \\
\hline$(3,50,0.95)$ & $3.00 \mid \mathbf{0 . 0 8 4}$ & 2.42 & $2.56 \times 10^{-3}$ & $0.61 \mid \mathbf{0 . 3 4}$ \\
\hline$(3,50,0.9)$ & $2.98 \mid \mathbf{0 . 0 8 3}$ & 2.41 & $1.97 \times 10^{-3}$ & $0.66 \mid \mathbf{0 . 3 8}$ \\
\hline$(3,100,0.95)$ & $5.81 \mid \mathbf{0 . 1 1}$ & 3.38 & $1.24 \times 10^{-3}$ & $1.81 \mid \mathbf{0 . 9 5}$ \\
\hline$(3,100,0.9)$ & $5.77 \mid \mathbf{0 . 1 1}$ & 3.37 & $7.00 \times 10^{-4}$ & $1.88 \mid \mathbf{0 . 9 5}$ \\
\hline
\end{tabular}

\section{B. DOAS}

In this subsection, we consider wavelength misalignment problem in different optical absorption spectroscopy analysis (DOAS). More specifically, this problem can be characterized as: $\boldsymbol{J}(\lambda)=\sum_{j=1}^{M} a_{j} \mathbf{y}_{j}\left(\lambda+v_{j}(\lambda)\right)+\boldsymbol{\eta}(\lambda) . \boldsymbol{J}(\lambda)$ presents the data and $\mathbf{y}_{j}\left(\lambda+v_{j}(\lambda)\right)$ denotes the reference spectra at the deformed wavelength $\lambda+v_{j}(\lambda)$ where $v_{j}(\cdot)$ denotes the deformations. The noise $\boldsymbol{\eta}(\lambda)$ are given at the wavelength $\lambda$ and $\left\{a_{j}\right\}_{j=1}^{M}$ are coefficients.

In our experiments, we generate a dictionary for three reference gases $(M=3)$ : $\mathrm{HONO}, \mathrm{NO} 2$ and $\mathrm{O} 3$, and then deform each with a set of linear functions, i.e., $v_{j}(\lambda)=$ $p_{j} \lambda+q_{j}$. We use $B_{j}(j=1, \cdots, M)$ to denote a matrix with each column be deformed basis, i.e.. $\mathbf{y}_{j}\left(\lambda+p_{k} \lambda+q_{\ell}\right)$ $(k=1, \cdots, K ; \quad \ell=1, \cdots, L)$ and $\mathbf{y}_{j} \in \mathbb{R}^{1024} ; p_{k}=$ $-1.01+0.01 k, q_{\ell}=-1.1+0.1 \ell$. By setting $K=L=21$, there are total of 441 linearly deformed references for each of the three groups.

We generate the dictionary by imitating the relative magnitudes of a real DOAS dataset [32] with a normalization to the dictionary. Then, to generate the data $a_{j}$, we randomly pick up one entry with random magnitudes whose mean values are $1,0.1,2$ for $\mathrm{HONO}, \mathrm{NO} 2$ and $\mathrm{O} 3$, respectively. Finally, the synthetic data $\mathcal{J}(\lambda)$ is generated by adding zero mean Gaussian noise. We consider five different noise levels, and its standard deviations (std) are $0,1 e-3,5 e-3,1 e-2,5 e-2$, respectively.

We solve the wavelength misalignment by considering the following model:

$\min _{\left\{\mathbf{x}_{j}\right\}_{j}} \frac{1}{2}\left\|\boldsymbol{J}-\left[B_{1}, \cdots, B_{M}\right]\left[\begin{array}{c}\mathbf{x}_{1} \\ \vdots \\ \mathbf{x}_{M}\end{array}\right]\right\|^{2}+\gamma \sum_{j=1}^{M} R\left(\mathbf{x}_{j}\right)$,

where $R(\cdot)$ represents the regularization function, and $\mathbf{x}_{j} \in$ $\mathbb{R}^{441}(j=1,2,3)$. We test 32 on different regularization functions to enforce sparsity. In particular, we set $R(\mathbf{x})=$ $\iota_{\mathbb{R}_{+}^{n}}(\mathbf{x}),\|\mathbf{x}\|_{1}+\iota_{\mathbb{R}_{+}^{n}}(\mathbf{x}),\|\mathbf{x}\|_{1}-\|\mathbf{x}\|_{2},\|\mathbf{x}\|^{1 / 2}, \frac{\|\mathbf{x}\|_{1}}{\|\mathbf{x}\|_{2}}+\iota_{\mathbb{R}_{+}^{n}}(\mathbf{x})$ in (32), respectively. We refer to these models as non-negative
TABLE II

RECONSTRUCTED ERROR (err $\left.=\left\|\hat{\mathbf{x}}-\mathbf{x}^{*}\right\|_{2}\right)$ FOR DOAS. FOR NOISELESS CASE $(s t d=0), \mathrm{ADMM}_{p}^{+}$PERFORMS THE SECOND BEST TO NNLS; FOR NOISY CASES ( std $\neq 0$ ), ADMM $_{p}^{+}$ALWAYS ACHIEVES THE HIGHEST ACCURACY AMONG THOSE ALGORITHMS.

\begin{tabular}{ccccccc}
\hline std & NNLS & NNL1 & $L_{1}-L_{2}$ & $L_{1 / 2}$ & \multicolumn{2}{c}{$\left(L_{1} / L_{2}\right)^{+}$} \\
\cline { 6 - 7 } & & & & & $\mathrm{ADMM}_{p}^{+}$ & SGPM \\
\hline 0 & $7.72 \mathrm{e}-16$ & $2.70 \mathrm{e}-03$ & $5.21 \mathrm{e}-05$ & $3.60 \mathrm{e}-03$ & $\mathbf{2 . 5 8 e - 0 5}$ & $4.80 \mathrm{e}-02$ \\
0.001 & $4.91 \mathrm{e}-03$ & $7.95 \mathrm{e}-03$ & $8.76 \mathrm{e}-04$ & $2.41 \mathrm{e}-02$ & $\mathbf{4 . 3 7 e - 0 4}$ & $7.60 \mathrm{e}-02$ \\
0.005 & $3.26 \mathrm{e}-02$ & $1.92 \mathrm{e}-02$ & $3.05 \mathrm{e}-03$ & $6.95 \mathrm{e}-02$ & $\mathbf{2 . 0 3 e - 0 3}$ & $3.75 \mathrm{e}-01$ \\
0.01 & $1.46 \mathrm{e}-01$ & $1.61 \mathrm{e}-01$ & $4.90 \mathrm{e}-03$ & $1.04 \mathrm{e}-01$ & $\mathbf{4 . 2 5 e - 0 3}$ & $3.84 \mathrm{e}-01$ \\
0.05 & $1.73 \mathrm{e}-01$ & $1.75 \mathrm{e}-01$ & $2.39 \mathrm{e}-02$ & $1.30 \mathrm{e}-01$ & $\mathbf{2 . 0 0 e - 0 2}$ & $5.67 \mathrm{e}-01$ \\
\hline
\end{tabular}

TABLE III

COMPUTATIONAL TIME (S) FOR DOAS UNDER DIFFERENT NOISY LEVEL. FOR SOLVING $\left(L_{1} / L_{2}\right)^{+}$, ADMM $_{p}^{+}$REDUCES COMPUTATIONAL TIME BY ABOUT $95 \% \sim 99 \%$ COMPARED TO SGPM.

\begin{tabular}{ccccccr}
\hline std & NNLS & NNL1 & $L_{1}-L_{2}$ & $L_{1 / 2}$ & \multicolumn{2}{c}{$\left(L_{1} / L_{2}\right)^{+}$} \\
\cline { 5 - 6 } & & & & & $\mathrm{ADMM}_{p}^{+}$ & SGPM \\
\hline 0 & 0.021 & 8.05 & 13.30 & 0.10 & 5.66 & 3110.00 \\
0.001 & 0.047 & 8.19 & 35.60 & 0.11 & 6.59 & 3030.00 \\
0.005 & 0.016 & 7.77 & 28.10 & 0.13 & 7.17 & 437.00 \\
0.01 & 0.057 & 8.50 & 33.90 & 0.13 & 8.34 & 167.00 \\
0.05 & 0.052 & 8.82 & 70.90 & 0.13 & 4.00 & 276.00 \\
\hline
\end{tabular}

least square (NNLS), non-negative unconstrained $L_{1}$ (NNL1), $L_{1}-L_{2}, L_{1 / 2},\left(L_{1} / L_{2}\right)^{+}$. For 32 with $\left(L_{1} / L_{2}\right)^{+}$regularizer, we adopt $\mathrm{ADMM}_{p}^{+}$and SGPM to solve it. For $L_{1}-L_{2}$, we use Algorithm 1 in [33]. For NNLS, we use MATLAB's Isqnonneg function. As for NNL1, we solve it by ADMM and for $L_{1 / 2}$, we solve it by [34]. For all these methods, we use the default setting.

Tables II and III show the errors (err $\left.=\left\|\hat{\mathbf{x}}-\mathbf{x}^{*}\right\|_{2}\right)$ between the reconstructed vectors and the ground-truth, and computational time (Time (s)) under different amounts of noise, respectively. Each recorded value is the average of 20 random realizations. In Fig. 2, the ground truth and the error vectors of these comparing algorithms defined by the constructed signals minus the true signal are presented in Plots (a) and (b), for the scenario of std $=0.05$. All the results demonstrate that $\mathrm{ADMM}_{p}^{+}$is comparable to NNLS in terms of accuracy for noiseless data, and even more accurate than NNLS for noisy cases. In comparison with NNL1, $L_{1}-L_{2}$, $\mathrm{ADMM}_{p}^{+}$also ends up with much higher accuracy and takes less time. In contrast with $L_{1 / 2}, \mathrm{ADMM}_{p}^{+}$converges to a much more accurate solution while consuming a bit more time. Besides, for solving the same $\left(L_{1} / L_{2}\right)^{+}$model, $\mathrm{ADMM}_{p}^{+}$ costs significantly less time than SGPM while still achieving a much more accurate solution.

\section{CONCLUSIONS}

We carry out a unified theoretical study on both $L_{1} / L_{2}$ minimization models, including the constrained and the unconstrained. First, we fill in the theoretical gap on exact recovery condition for the unconstrained model. Indeed, we provide a uniformed exact recovery condition both for the constrained and the unconstrained models. This condition turns to be more easily checkable, especially when $A$ is low rank. Second, for these $L_{1} / L_{2}$ minimization models, we prove that the existence of the globally optimal solution can be 
(a) Ground truth

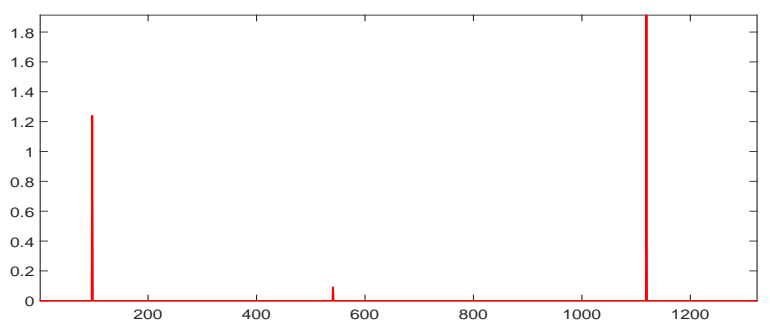

(b) $E R R=\hat{x}-x^{*}$

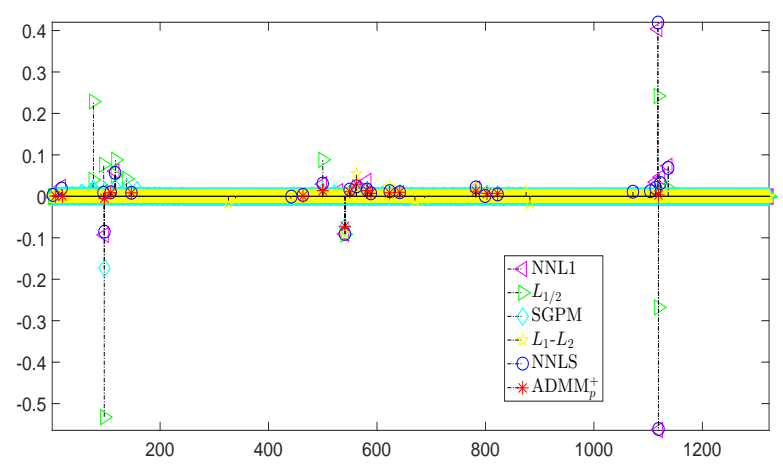

Fig. 1. Comparisons for NNLS, NNL1, $L_{1}-L_{2}, L_{1 / 2}$, SGPM and $\mathrm{ADMM}_{p}^{+}$ on DOAS data with additive noise ( $\mathrm{std}=5 e-2)$. (a) The ground truth with sparsity 3; (b) The error vectors from these comparing algorithms defined by the constructed signals minus the ground truth, and its nonzero numbers of these error vectors are $27,1323,1323,1323,1323,18$, respectively. The horizontal heavy yellow line surrounded by cyan are caused by the fulldimension error vectors from $L_{1}-L_{2}$, SGPM, NNL1, $L_{1 / 2}$. The deviation of $\mathrm{ADMM}_{p}^{+}$is much smaller than the others. $\mathrm{ADMM}_{p}^{+}$achieves the best recovery quality in the sense of highest accuracy and sparsity.

guaranteed by the $s$-spherical section property of the null space of the matrix $A$. Third, we derive a closed-form solution of the proximal operator of $\left(L_{1} / L_{2}\right)^{+}$. Equipped with this, we propose a specific splitting scheme $\left(\mathrm{ADMM}_{p}^{+}\right)$to solve the unconstrained $\left(L_{1} / L_{2}\right)^{+}$model. We establish its global convergence to a d-stationary (sharpest stationary) solution by verifying the KL property of the potential function. Local linear convergence of $\mathrm{ADMM}_{p}^{+}$is also proved under certain conditions. Numerical simulations validate our analyses and demonstrate that $\mathrm{ADMM}_{p}^{+}$outperforms other state-of-the-art methods in sparse recovery.

\section{APPENDIX A}

\section{PROOF OF THEOREM 7}

Proof: First, define $r(\mathbf{x})=\gamma \frac{\|\mathbf{x}\|_{1}}{\|\mathbf{x}\|_{2}}$. Assume that $\left\{\mathbf{x}^{k}\right\}$ is a minimizing sequence of (3), i.e.,

$$
\lim _{k \rightarrow \infty} F\left(\mathbf{x}^{k}\right)=F^{*} .
$$

If the sequence $\left\{\mathrm{x}^{k}\right\}$ is bounded, then it has a subsequence $\left\{\mathbf{x}^{k_{j}}\right\}$ converging to some $\mathbf{x}^{*}$. Hence, $\mathbf{x}^{*}$ is an optimal solution of 3 . Otherwise, the sequence $\left\{\mathbf{x}^{k}\right\}$ is unbounded, i.e., $\left\|\mathbf{x}^{k}\right\| \rightarrow \infty$ as $k \rightarrow \infty$. Since the sequence of $\left\{r\left(\mathbf{x}^{k}\right)\right\}$ is bounded below and $F^{*}$ is finite, it leads to the sequence of $\left\{\frac{1}{2}\left\|A \mathbf{x}^{k}-\mathbf{b}\right\|_{2}^{2}\right\}$ is bounded above. It implies that the subsequence of $\left\{A \mathbf{x}^{k}\right\}$ is bounded. Since $\left\{A \mathbf{x}^{k}\right\}$ is bounded, it has a sequence that converges to $\mathbf{y}^{*}$. Without loss of generality, we assume that $A \mathbf{x}^{k} \rightarrow \mathbf{y}^{*}$. Let $\mathcal{I}=\left\{j:\left\{x_{j}^{k}\right\}\right.$ is bounded $\}$. Then, it follows that $\left\{A_{\mathcal{I}} \mathbf{x}_{\mathcal{I}}^{k}\right\}$ is bounded. This together with boundedness of $\left\{A \mathbf{x}^{k}\right\}$ implies that $\left\{A_{\mathcal{I}^{c}} \mathbf{x}_{\mathcal{I}^{c}}^{k}\right\}$ is also bounded. Next, for each $k$, we consider the following linear system,

$$
A_{\mathcal{I}^{c}} \mathbf{y}^{k}=A_{\mathcal{I}^{c}} \mathbf{x}_{\mathcal{I}^{c}}^{k}, \mathbf{y}^{k} \in \mathcal{Y},
$$

where $\mathcal{Y}:=\mathbb{R}^{\sharp\left(\mathcal{I}^{c}\right)}$ if $\mathcal{X}=\mathbb{R}^{n}$ and $\mathcal{Y}:=\mathbb{R}_{+}^{\sharp\left(\mathcal{I}^{c}\right)}$ if $\mathcal{X}=$ $\mathbb{R}_{+}^{n}$. Obviously, the solution set of the above linear system is nonempty due to at least one solution $\mathbf{y}^{k}=\mathbf{x}_{\mathcal{I}^{c}}^{k}$ for each $k$. Using Hoffman's Error Bound [35], there exist a vector $\mathbf{y}^{k}$ satisfying $A_{\mathcal{I}^{c}} \mathbf{y}^{k}=A_{\mathcal{I}^{c}} \mathbf{x}_{\mathcal{I}^{c}}^{k}$ and a constant $\zeta>0$ depending only on $A_{\mathcal{I}^{c}}$ such that

$$
\left\|\mathbf{y}^{k}\right\| \leq \zeta\left\|A_{\mathcal{I}^{c}} \mathbf{x}_{\mathcal{I}^{c}}^{k}\right\| .
$$

By setting $\hat{\mathbf{x}}^{k}=\left(\mathbf{x}_{\mathcal{I}}^{k}, \mathbf{y}^{k}\right)$, it leads to $A \hat{\mathbf{x}}^{k}=A \mathbf{x}^{k}$ and $\hat{\mathbf{x}}^{k} \rightarrow$ $\mathbf{x}^{*}$ for convenience due to its boundedness of $\left\{\hat{\mathbf{x}}^{k}\right\}$. Obviously, $\mathbf{y}^{*}=A \mathbf{x}^{*}$ thanks to $A \hat{\mathbf{x}}^{k_{j}}=A \mathrm{x}^{k_{j}}$. In the following, we divide into two cases to verify.

Case 1. If there exists two subsequences $\left\{\mathbf{x}^{k_{j}}\right\}$ and $\left\{\hat{\mathbf{x}}^{k_{j}}\right\}$ such that

$$
\frac{\left\|\mathbf{x}^{k_{j}}\right\|_{1}}{\left\|\mathbf{x}^{k_{j}}\right\|_{2}} \geq \frac{\left\|\hat{\mathbf{x}}^{k_{j}}\right\|_{1}}{\left\|\hat{\mathbf{x}}^{k_{j}}\right\|_{2}}, \forall j .
$$

Since $A \hat{\mathbf{x}}^{k_{j}}=A \mathbf{x}^{k_{j}}$, we have that

$$
F\left(\mathbf{x}^{k_{j}}\right) \geq F\left(\hat{\mathbf{x}}^{k_{j}}\right) .
$$

By using $\hat{\mathbf{x}}^{k_{j}} \rightarrow \mathrm{x}^{*}$ and $F$ lower semi-continuous, it yields that

$$
\varliminf_{j \rightarrow \infty} F\left(\hat{\mathbf{x}}^{k_{j}}\right) \geq F\left(\mathbf{x}^{*}\right) .
$$

On the other hand,

$F^{*}=\lim _{k \rightarrow \infty} F\left(\mathbf{x}^{k}\right)=\lim _{j \rightarrow \infty} F\left(\mathbf{x}^{k_{j}}\right) \geq \lim _{j \rightarrow \infty} F\left(\hat{\mathbf{x}}^{k_{j}}\right) \geq F\left(\mathbf{x}^{*}\right)$.

Invoking the definition of $F^{*}$, it leads to $F\left(\mathbf{x}^{*}\right)=F^{*}$ and $\mathbf{x}^{*}$ is an optimal solution.

Case 2. If there does not exist such two sequences $\left\{\mathbf{x}^{k_{j}}\right\}$ and $\left\{\hat{\mathbf{x}}^{k_{j}}\right\}$ satisfying 34 , it implies there exists an index $K$ such that

$$
\frac{\left\|\mathbf{x}^{k}\right\|_{1}}{\left\|\mathbf{x}^{k}\right\|_{2}}<\frac{\left\|\hat{\mathbf{x}}^{k}\right\|_{1}}{\left\|\hat{\mathbf{x}}^{k}\right\|_{2}}, \quad \forall k \geq K .
$$

Next, we further divide into two cases to verify.

(a) Suppose that $\mathbf{y}^{*} \in \operatorname{Proj}_{(A \mathcal{X})}(\mathbf{b})$. Then, the solution set of 10] with $\mathbf{c}=\mathbf{y}^{*}$ is nonempty due to $f_{p c}^{*}\left(\mathbf{y}^{*}\right)<f_{d c}^{*}$. We assume that $\hat{\mathbf{x}}$ is an optimal solution of (10) with $\mathbf{c}=\mathbf{y}^{*}$. Since 33 and $A \mathbf{x}^{k} \rightarrow \mathbf{y}^{*}$, then

$$
\lim _{k \rightarrow \infty} \frac{\left\|\mathbf{x}^{k}\right\|_{1}}{\left\|\mathbf{x}^{k}\right\|_{2}}=\left(F^{*}-\frac{1}{2}\left\|\mathbf{y}^{*}-\mathbf{b}\right\|_{2}^{2}\right)<+\infty .
$$

Thus, $\lim _{k \rightarrow \infty} \frac{\left\|\mathbf{x}^{k}\right\|_{1}}{\left\|\mathbf{x}^{k}\right\|_{2}}$ exits. Next, we verify that

$$
\lim _{k \rightarrow \infty} \frac{\left\|\mathbf{x}^{k}\right\|_{1}}{\left\|\mathbf{x}^{k}\right\|_{2}} \leq \frac{\|\hat{\mathbf{x}}\|_{1}}{\|\hat{\mathbf{x}}\|_{2}}=f_{p c}^{*}\left(\mathbf{y}^{*}\right) .
$$


Suppose not, i.e., $\lim _{k \rightarrow \infty} \frac{\left\|\mathbf{x}^{k}\right\|_{1}}{\left\|\mathbf{x}^{k}\right\|_{2}}>\frac{\|\hat{\mathbf{x}}\|_{1}}{\|\hat{\mathbf{x}}\|_{2}}$, it implies that $F(\hat{\mathbf{x}})<F^{*}$ since $A \hat{\mathbf{x}}=A \mathbf{x}^{*}$. It contradicts to the definition of $F^{*}$. Thus, 35 holds. We define $\tilde{\mathbf{x}}^{k}:=\frac{\mathbf{x}^{k}}{\left\|\mathbf{x}^{k}\right\|_{2}}$. Taking $k \rightarrow \infty$,

$$
A \mathbf{x}^{k} \rightarrow \mathbf{y}^{*} \Rightarrow A \tilde{\mathbf{x}}^{k} \rightarrow \mathbf{0},
$$

since $\left\|\mathbf{x}^{k}\right\|_{2} \rightarrow \infty$. Since $\tilde{\mathbf{x}}^{k}$ is bounded, it has a sequence $\tilde{\mathbf{x}}^{k_{j}} \rightarrow \overline{\mathbf{x}}$ where $\overline{\mathbf{x}}$ is satisfied with $\overline{\mathbf{x}} \in \mathcal{F}_{0}$.

$$
\lim _{k \rightarrow \infty} \frac{\left\|\mathbf{x}^{k}\right\|_{1}}{\left\|\mathbf{x}^{k}\right\|_{2}}=\frac{\|\overline{\mathbf{x}}\|_{1}}{\|\overline{\mathbf{x}}\|_{2}} \geq f_{d c}^{*}>f_{p c}^{*}\left(\mathbf{y}^{*}\right),
$$

where the first inequality is due to $\overline{\mathrm{x}} \in \mathcal{F}_{0}$ and the last inequality follows from $\mathbf{y}^{*} \in \operatorname{Proj}_{A \mathcal{X}}(\mathbf{b})$. Note that the above inequality contradicts to 35.

(b) Suppose that $\mathbf{y}^{*} \notin \operatorname{Proj}_{A \mathcal{X}}(\mathbf{b})$. Then, we pick up one vector $\hat{\mathbf{y}} \in \operatorname{Proj}_{A \mathcal{X}}(\mathbf{b})$. Consider the constrained problem 10 with $\mathbf{c}:=\hat{\mathbf{y}}$. Since $f_{p c}^{*}(\hat{\mathbf{y}})<f_{d c}^{*}$, the solution set of (10) with $\mathbf{c}:=\hat{\mathbf{y}}$ is nonempty due to Corollary 2. We assume that $\mathbf{x}_{0}$ is an optimal solution of $(10)$ with $\mathbf{c}:=\hat{\mathbf{y}}$. It follows that (36) that

$$
\lim _{k \rightarrow \infty} \gamma \frac{\left\|\mathbf{x}^{k}\right\|_{1}}{\left\|\mathbf{x}^{k}\right\|_{2}}=\gamma \frac{\|\overline{\mathbf{x}}\|_{1}}{\|\overline{\mathbf{x}}\|_{2}} \geq \gamma f_{d c}^{*}
$$

where the definition of $\overline{\mathbf{x}}$ is the same as Case (a). By noting $\hat{\mathbf{y}} \in \operatorname{Proj}_{A \mathcal{X}}(\mathbf{b})$ and $\mathbf{y}^{*} \in A \mathcal{X}$ since $A \mathbf{x}^{k} \in A \mathcal{X}$ and $A \mathbf{x}^{k} \rightarrow \mathbf{y}^{*}$, it leads to $\frac{1}{2}\|\hat{\mathbf{y}}-\mathbf{b}\|^{2} \leq \frac{1}{2} \| \mathbf{y}^{*}-$ $\mathbf{b} \|^{2}$. Thus, $\frac{1}{2}\left\|A \mathbf{x}_{0}-\mathbf{b}\right\|^{2} \leq \frac{1}{2}\left\|\mathbf{y}^{*}-\mathbf{b}\right\|^{2}$. By noting $r\left(\mathbf{x}_{0}\right)=\gamma f_{p c}^{*}(\hat{\mathbf{y}})<\gamma f_{d c}^{*}$, and combining with the above inequality, it yields that

$$
F\left(\mathbf{x}_{0}\right)<\gamma f_{d c}^{*}+\frac{1}{2}\left\|\mathbf{y}^{*}-\mathbf{b}\right\|^{2} .
$$

On the other hand, combining (37) with the fact of $A \mathbf{x}^{k} \rightarrow \mathbf{y}^{*}$, we have

$$
\begin{aligned}
& \gamma f_{d c}^{*}+\frac{1}{2}\left\|\mathbf{y}^{*}-\mathbf{b}\right\|^{2} \\
& \leq \lim _{k \rightarrow \infty}\left(\gamma \frac{\left\|\mathbf{x}^{k}\right\|_{1}}{\left\|\mathbf{x}_{k}\right\|_{2}}+\frac{1}{2}\left\|A \mathbf{x}^{k}-\mathbf{b}\right\|_{2}^{2}\right)=F^{*} .
\end{aligned}
$$

In view of (38) and the above inequality, it leads to $F\left(\mathbf{x}_{0}\right)<F^{*}$ which contradicts to the definition of $F^{*}$.

Therefore, the sequence $\left\{\mathbf{x}^{k}\right\}$ is bounded, and thus it has an accumulation point $\mathrm{x}^{*}$ which is an optimal solution of (3).

\section{APPENDIX B}

\section{Proof of THEOREM 13}

Proof: First, the sequence of $\left\{\mathbf{w}^{k}\right\}$ converges to a stationary point $\mathbf{w}^{\infty}$ due to Theorem 12 Next, we can show that $\operatorname{supp}\left(\mathbf{x}^{k}\right) \equiv \bar{\Lambda}$ when $k$ is sufficiently large due to $\mathbf{x}^{k} \rightarrow \mathbf{x}^{\infty}$. Consequently, $\mathbf{x}_{i}^{k}>0$ where $i \in \bar{\Lambda}$ when $k$ is sufficiently large due to $\mathcal{X}=\mathbb{R}_{+}^{n}$. Analogously, $\operatorname{supp}\left(\mathbf{y}^{k}\right) \equiv \bar{\Lambda}$ when $k$ is sufficiently large.

In the following, by defining

$$
\Pi=\left\{\mathbf{x} \in \mathbb{R}_{+}^{n} \mid \operatorname{supp}(\mathbf{x})=\bar{\Lambda}\right\},
$$

we will verify that there exists a scalar $\bar{\gamma}>0$ such that $F$ is a KL function with an exponent of $1 / 2$ restricted on $\Pi$ when $0<$ $\gamma<\bar{\gamma}$. In order to do so, we define the function $\tilde{F}: \mathbb{R}^{\alpha} \rightarrow \mathbb{R}$

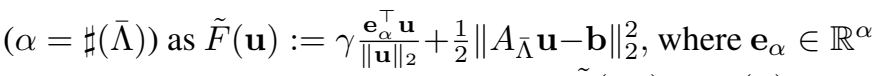
with each entry equal to 1 . Obviously, $\tilde{F}\left(\mathbf{x}_{\bar{\Lambda}}\right)=F(\mathbf{x})$ when $\mathbf{x} \in \Pi$. Define $\Pi_{\bar{\Lambda}}:=\left\{\mathbf{u} \in \mathbb{R}_{+}^{\alpha} \mid \mathbf{u}=\mathbf{x}_{\bar{\Lambda}}, \mathbf{x} \in \Pi\right\}$. $\tilde{F}$ is differentiable on the set $\Pi_{\bar{\Lambda}}$ and its gradient is

$\nabla \tilde{F}(\mathbf{u})=\gamma\left(\frac{\mathbf{e}_{\alpha}}{\|\mathbf{u}\|_{2}}-\frac{\|\mathbf{u}\|_{1}}{\|\mathbf{u}\|_{2}^{3}} \mathbf{u}\right)+A_{\bar{\Lambda}}^{\top}\left(A_{\bar{\Lambda}} \mathbf{x}_{\bar{\Lambda}}-\mathbf{b}\right), \forall \mathbf{u} \in \Pi_{\bar{\Lambda}}$.

Next, define the set $\tilde{\mathcal{X}}=\left\{\mathbf{x} \in \mathbb{R}_{+}^{n} \mid\left\|\mathbf{x}_{\bar{\Lambda}}\right\|_{2} \geq \epsilon_{0}\right\}$ where $0<\epsilon_{0}<\frac{1}{2}\left\|\mathbf{x}^{\infty}\right\|_{2}$. Obviously, $\mathbf{x}^{\infty} \in \tilde{\mathcal{X}}$. In the following, we show that there exists a parameter $\bar{\gamma}>0$ such that when $0<\gamma<\bar{\gamma}$, it holds that

$$
\begin{array}{r}
\left\|\nabla \tilde{F}\left(\mathbf{x}_{\bar{\Lambda}}\right)-\nabla \tilde{F}\left(\left(\mathbf{x}^{\infty}\right)_{\bar{\Lambda}}\right)\right\|_{2} \geq \hat{\tau}\left\|\mathbf{x}_{\bar{\Lambda}}-\left(\mathbf{x}^{\infty}\right)_{\bar{\Lambda}}\right\|_{2} \\
\forall \mathbf{x} \in \Pi \cap \tilde{\mathcal{X}},
\end{array}
$$

with $\hat{\tau}>0$.

First, by defining the function $g(\mathbf{u}):=\frac{\mathbf{e}_{\alpha}}{\|\mathbf{u}\|_{2}}-\frac{\|\mathbf{u}\|_{1}}{\|\mathbf{u}\|_{2}^{3}} \mathbf{u}$ and the set $\tilde{\mathcal{X}}_{\bar{\Lambda}}:=\left\{\mathbf{u} \in \mathbb{R}_{+}^{\alpha} \mid \mathbf{u}=\mathbf{x}_{\bar{\Lambda}}, \mathbf{x} \in \tilde{\mathcal{X}}\right\}$, some elementary calculations leads to that

$$
\|g(\mathbf{u})-g(\mathbf{v})\|_{2} \leq \tilde{L}\|\mathbf{u}-\mathbf{v}\|_{2} \quad \forall \mathbf{u}, \mathbf{v} \in \Pi_{\bar{\Lambda}} \cap \tilde{\mathcal{X}}_{\bar{\Lambda}},
$$

where $\tilde{L}:=\frac{6 \sqrt{\alpha}}{\epsilon_{0}^{2}}$. Next, we prove 40 . For any $\mathrm{x} \in \Pi \cap \tilde{\mathcal{X}}$, we have

$$
\begin{aligned}
& \left\|\nabla \tilde{F}\left(\mathbf{x}_{\bar{\Lambda}}\right)-\nabla \tilde{F}\left(\left(\mathbf{x}^{\infty}\right)_{\bar{\Lambda}}\right)\right\|_{2} \\
& \quad \geq\left\|A_{\Lambda}^{\top} A_{\Lambda}\left(\mathbf{x}_{\bar{\Lambda}}-\left(\mathbf{x}^{\infty}\right)_{\bar{\Lambda}}\right)\right\|_{2}-\gamma\left\|g\left(\mathbf{x}_{\bar{\Lambda}}\right)-g\left(\left(\mathbf{x}^{\infty}\right)_{\bar{\Lambda}}\right)\right\|_{2} \\
& \quad \geq \delta\left\|\mathbf{x}_{\bar{\Lambda}}-\left(\mathbf{x}^{\infty}\right)_{\bar{\Lambda}}\right\|_{2},
\end{aligned}
$$

where $\delta=\sigma_{\min }\left(A_{\bar{\Lambda}}^{\top} A_{\bar{\Lambda}}\right)-\gamma \tilde{L}$ and $\sigma_{\min }(\cdot)$ represents the smallest eigenvalue. Set $\bar{\gamma}=\frac{\sigma_{\min }\left(A_{\bar{\Lambda}}^{\top} A_{\bar{\Lambda}}\right)}{\tilde{L}}$. Consequently, the inequality 40 holds with $\hat{\tau}:=\sigma_{\min }\left(A_{\bar{\Lambda}}^{\top} A_{\bar{\Lambda}}\right)-\gamma \tilde{L}>0$ when $0<\gamma<\bar{\gamma}$.

Furthermore, since the function $g(\mathbf{u})$ is Lipschitz continuous with constant $\tilde{L}$ on the set of $\Pi_{\bar{\Lambda}} \cap \tilde{\mathcal{X}}_{\bar{\Lambda}}$, it leads to

$$
\|\nabla \tilde{F}(\mathbf{u})-\nabla \tilde{F}(\mathbf{v})\| \leq \hat{L}\|\mathbf{u}-\mathbf{v}\|, \quad \forall \mathbf{u}, \mathbf{v} \in \Pi_{\bar{\Lambda}} \cap \tilde{\mathcal{X}}_{\bar{\Lambda}},
$$

where $\hat{L}:=\gamma \tilde{L}+\sigma_{\max }\left(A_{\bar{\Lambda}}^{\top} A_{\bar{\Lambda}}\right)$.

Since $\mathbf{x}^{\infty}$ is satisfying (5), it leads to that

$$
\nabla \tilde{F}\left(\left(\mathbf{x}^{\infty}\right)_{\bar{\Lambda}}\right)=\mathbf{0} .
$$

Consequently, for any $\mathrm{x} \in \Pi \cap \tilde{\mathcal{X}}$, we have

$$
\begin{aligned}
& \left|F(\mathbf{x})-F\left(\mathbf{x}^{\infty}\right)\right|=\left|\tilde{F}\left(\mathbf{x}_{\bar{\Lambda}}\right)-\tilde{F}\left(\left(\mathbf{x}^{\infty}\right)_{\bar{\Lambda}}\right)\right| \\
& \quad \leq \frac{\hat{L}}{2}\left\|\mathbf{x}_{\bar{\Lambda}}-\left(\mathbf{x}^{\infty}\right)_{\bar{\Lambda}}\right\|^{2} .
\end{aligned}
$$

By combining (42), 40] and 441, we have that

$$
\left|F(\mathbf{x})-F\left(\mathbf{x}^{\infty}\right)\right| \leq \tilde{c}\left\|\nabla \tilde{F}\left(\mathbf{x}_{\bar{\Lambda}}\right)\right\|^{2}, \quad \forall \mathbf{x} \in \Pi \cap \tilde{\mathcal{X}},
$$

with $\tilde{c}:=\frac{\hat{L}}{2(\hat{\tau})^{2}}$. Finally, we choose a neighborhood $U\left(\mathbf{x}^{\infty}\right)$ of $\mathbf{x}^{\infty}$ such that $U\left(\mathbf{x}^{\infty}\right) \subseteq \tilde{\mathcal{X}}$ and define $\mathcal{B}$ as

$\mathcal{B}=\left\{\mathbf{x} \in \mathbb{R}_{+}^{n} \mid \mathbf{x} \in \Pi \cap U\left(\mathbf{x}^{\infty}\right), F\left(\mathbf{x}^{\infty}\right)<F(\mathbf{x})<F\left(\mathbf{x}^{\infty}\right)+\nu\right\}$.

Thus, it leads to

$$
\begin{aligned}
& \inf _{\mathbf{x} \in \mathcal{B}}\left\|\nabla \tilde{F}\left(\mathbf{x}_{\bar{\Lambda}}\right)\right\|^{2}=\inf _{\substack{\xi \in \partial F(\mathbf{x}) \\
\mathbf{x} \in \mathcal{B}}}\left\|\left.\xi\right|_{\bar{\Lambda}}\right\|^{2} \\
& \leq \inf _{\substack{\xi \in \partial F(\mathbf{x}) \\
\mathbf{x} \in \mathcal{B}}}\|\xi\|^{2}=\operatorname{dist}^{2}(\mathbf{0}, \partial F(\mathbf{x})) .
\end{aligned}
$$


By combining the above inequality with (43), one has that $F$ is a $\mathrm{KL}$ function restricted on $\Pi$ with an exponent of $1 / 2$.

Finally, since both of $\mathbf{x}^{k}$ and $\mathbf{y}^{k}$ enter into the set of $\Pi$ when $k$ is sufficiently large, we have that $\mathcal{T}(\mathbf{x}, \mathbf{y})$ is a KL function with an exponent of $1 / 2$ at $\left(\mathbf{x}^{\infty}, \mathbf{x}^{\infty}\right)$ restricted on $\Pi \times \Pi$ by following the proof to [36. Theorem 3.6]. The remaining proof follows directly from [37] and thus omitted here.

\section{ACKNOWLEDGMENTS}

We thank Dr. Penghang Yin of the Department of Mathematics and Statistics, University at Albany, The State University of New York, for providing us with Matlab codes of DOAS.

\section{REFERENCES}

[1] B. K. Natarajan, "Sparse approximate solutions to linear systems," SIAM J. Comput., vol. 24, no. 2, pp. 227-234, 1995.

[2] S. S. Chen, D. L. Donoho, and M. A. Saunders, "Atomic decomposition by basis pursuit," SIAM J. Sci. Comput., vol. 20, no. 1, pp. 33-61, 1998.

[3] E. J. Candes, J. K. Romberg, and T. Tao, "Stable signal recovery from incomplete and inaccurate measurements," Comm. Pure Appl. Math., vol. 59, no. 8, pp. 1207-1223, 2006.

[4] J. Kim, J. Wang, and B. Shim, "Optimal restricted isometry condition of normalized sampling matrices for exact sparse recovery with orthogonal least squares," IEEE Trans. Signal Process., vol. 69, pp. 1521-1536, 2021.

[5] D. L. Donoho and X. Huo, "Uncertainty principles and ideal atomic decomposition," IEEE Trans. Inform. Theory, vol. 47, no. 7, pp. 28452862, 2001.

[6] A. Cherni, E. Chouzenoux, L. Duval, and J.-C. Pesquet, "SPOQ $\ell_{p}$ over- $\ell_{q}$ regularization for sparse signal recovery applied to mass spectrometry," IEEE Trans. Signal Process., vol. 68, pp. 6070-6084, 2020.

[7] L. Wu, Z. Sun, and D.-H. Li, "A barzilai-borwein-like iterative half thresholding algorithm for the $\ell_{1 / 2}$ regularized problem," J. Sci. Comput., vol. 67, no. 2, pp. 581-601, 2016.

[8] P. Yin, E. Esser, and J. Xin, "Ratio and difference of $\ell_{1}$ and $\ell_{2}$ norms and sparse representation with coherent dictionaries," Comm. Info. Systems, vol. 14, no. 2, pp. 87-109, 2014.

[9] P. O. Hoyer, "Non-negative sparse coding," in Proc. IEEE Workshop on Neural Networks for Signal Processing. IEEE, 2002, pp. 557-565.

[10] H. Ji, J. Li, Z. Shen, and K. Wang, "Image deconvolution using a characterization of sharp images in wavelet domain," Appl. and Comput. Harmon. A., vol. 32, no. 2, pp. 295-304, 2012.

[11] D. Krishnan, T. Tay, and R. Fergus, "Blind deconvolution using a normalized sparsity measure," in Proc. IEEE Comput. Soc. Conf. Comput. Vis. Pattern Recognit., 2011, pp. 233-240.

[12] C. Wang, M. Tao, J. G. Nagy, and Y. Lou, "Limited-angle CT reconstruction via the $\mathrm{L}_{1} / \mathrm{L}_{2}$ minimization," SIAM J. Imaging Sci., vol. 14, no. 2, pp. 749-777, 2021.

[13] E. Esser, Y. Lou, and J. Xin, "A method for finding structured sparse solutions to nonnegative least squares problems with applications," SIAM J. Imaging Sci., vol. 6, no. 4, pp. 2010-2046, 2013.

[14] Y. Rahimi, C. Wang, H. Dong, and Y. Lou, "A scale-invariant approach for sparse signal recovery," SIAM J. Sci. Comput., vol. 41, no. 6, pp. A3649-A3672, 2019.

[15] M. Tao, "Minimization of L1 over L2 for sparse signal recovery with convergence guarantee," available at http://www.optimization-online. org/DB_FILE/2020/10/8064.pdf

[16] C. Wang, M. Yan, Y. Rahimi, and Y. Lou, "Accelerated schemes for the $\mathrm{L}_{1} / \mathrm{L}_{2}$ minimization," IEEE Trans. Signal Process., vol. 68, pp. 2660 2669, 2020.

[17] L. Zeng, P. Yu, and T. K. Pong, "Analysis and algorithms for some compressed sensing models based on L1/L2 minimization," SIAM J. Optim., vol. 31, no. 2, pp. 1576-1603, 2021.

[18] G. Li and T. K. Pong, "Global convergence of splitting methods for nonconvex composite optimization," SIAM J. Optim., vol. 25, no. 4, pp. 2434-2460, 2015

[19] M. Hong, Z.-Q. Luo, and M. Razaviyayn, "Convergence analysis of alternating direction method of multipliers for a family of nonconvex problems," SIAM J. Optim., vol. 26, no. 1, pp. 337-364, 2016.
[20] J.-S. Pang and M. Tao, "Decomposition methods for computing directional stationary solutions of a class of nonsmooth nonconvex optimization problems," SIAM J. Optim., vol. 28, no. 2, pp. 1640-1669, 2018.

[21] T.-H. Chang, M. Hong, W.-C. Liao, and X. Wang, "Asynchronous distributed ADMM for large-scale optimization - Part I: Algorithm and convergence analysis," IEEE Trans. Signal Process., vol. 64, pp. 3118 3130, 2016.

[22] F. H. Clarke, Optimization and Nonsmooth Analysis. Classical Appl. Math. Society for Industrial and Applied Mathematics, Philadelphia, PA, 1990, vol. 5 .

[23] J. Li, A. M.-C. So, and W.-K. Ma, "Understanding notions of stationarity in nonsmooth optimization: A guided tour of various constructions of subdifferential for nonsmooth functions," IEEE Signal Proc. Mag., vol. 37, no. 5, pp. 18-31, 2020.

[24] J.-S. Pang, M. Razaviyayn, and A. Alvarado, "Computing B-stationary points of nonsmooth DC programs," Math. Oper. Res., vol. 42, no. 1, pp. $95-118,2017$.

[25] H. Dong and M. Tao, "On the linear convergence to weak/standard dstationary points of DCA-based algorithms for structured nonsmooth dc programming," J. Optim. Theory Appl., vol. 189, pp. 190-220, 2021.

[26] J. Bolte, A. Daniilidis, and A. Lewis, "The łojasiewicz inequality for nonsmooth subanalytic functions with applications to subgradient dynamical systems," SIAM J. Optim., vol. 17, no. 4, pp. 1205-1223, 2007.

[27] S. A. Vavasis, "Derivation of compressive sensing theorems from the spherical section property," University of Waterloo, 2009.

[28] Y. Zhang, "Theory of compressive sensing via $\mathrm{L}_{1}$-minimization: A nonrip analysis and extensions," J. Oper. Res. Soc. China, vol. 1, no. 1, pp. 79-105, 2013.

[29] W. Hare and C. Sagastizábal, "Computing proximal points of nonconvex functions," Math. Program., vol. 116, no. 1, pp. 221-258, 2009.

[30] C. Yang, Y. Gu, B. Chen, H. Ma, and H. C. So, "Learning proximal operator methods for nonconvex sparse recovery with theoretical guarantee," IEEE Trans. Signal Process., vol. 68, pp. 5244-5259, 2020.

[31] J. Bochnak, M. Coste, and M. F. Roy, Real Algebraic Geometry. Ergeb. Math. Grenzgeb. 36, Springer-Verlag, Berlin, 1998.

[32] B. Finlayson-Pitts, "Unpublished data," 2000, provided by L. M. Wingen.

[33] Y. Lou, S. Osher, and J. Xin, "Computational aspects of constrained $L_{1}-L_{2}$ minimization for compressive sensing," in Model. Comput. \& Optim. Inf. Syst. \& Manage. Sci., Advances in Intelligent Systems and Computing, 2015, vol. 359, pp. 169-180.

[34] M.-J. Lai, Y. Xu, and W. Yin, "Improved iteratively reweighted least squares for unconstrained smoothed $\ell_{q}$ minimization," SIAM J. Numer. Anal., vol. 51, no. 2, pp. 927-957, 2013.

[35] A. J. Hoffman, "On approximate solutions of systems of linear inequalities,” J. Res. Nat. Bur. Standards, vol. 49, no. 4, pp. 263-265, 1952.

[36] G. Li and T. K. Pong, "Calculus of the exponent of kurdyka-łojasiewicz inequality and its applications to linear convergence of first-order methods," Found. Comput. Math., vol. 18, no. 5, pp. 1199-1232, 2018.

[37] H. Attouch and J. Bolte, "On the convergence of the proximal algorithm for nonsmooth functions involving analytic features," Math. Program., vol. 116, no. 1, pp. 5-16, 2009. 